[Aus dem hygienischen Institut der Universität Halle a/S.]

(Direktor: Geh. Med.-Rat Prof. Dr. C. Fraenkel.)

\title{
Trinkwasserbeurteilung und Trinkwasserversorgung ${ }^{1}$ bei der Feldarmee.
}

Vortrag, gehalten vor der Vereinigung der Sanitätsoffiziere des IV. Armeekorps in Magdeburg.

Von

Oberstabsarzt Baehr,

Regimentsurat des FüBilierregiments Generalfeldmarschall Graf Blumeathal (Magdebargischen) Nr. 36.

Die Schlagfertigkeit einer Armee hängt wesentlich ab von der Fernhaltung oder Unterdrückung gefahrdrohender Heeresseuchen.

Die Kriegsgeschichte ist reich an Beispielen, welche die Wahrheit dieses Satzes bestätigen. So endete, wie Cassius Dio berichtet, im Jahre 24 v. Chr. der Heereszug des rồmischen Feldherrn Aelius Gallus, nachdem zuvor Ägypten unterworfen worden war, in dem gesegneten Arabien kläglich, da der größte Teil seines Heeres durch Krankheiten zugrunde ging. Ebenso wurden die Alemannen im Jahre 555 n. Chr. nach der Eroberung Roms durch tödliche Erkrankungen, welche ihre Reihen lichteten, zum Rückzuge gezwungen. Das Heer der Kreuzfahrer unter Gottfried von Bouillon, welches beim Aufbruch am 15. VIII. 1096 700000 Mann stark war, war durch Krankheiten, Strapazen und Seuchen beim Übergang nach Kleinasien Anfang Mai 1097 bis auf 400000 Mann zusammengeschmolzen und langte nach weiteren Verlusten durch Seuchen mit nur 20000 Mann am 6. Mai 1099 vor Jerusalem an. Die Armée

1 Die Arbeit: „Zur Sicherstellung der Trinkwasserversorgung im Felde von Oberstabsarzt Dr. Musehold und Stabsarzt Dr. Bisch off " in der Gedenkschrift für v. Leuthold ist mir erst nach dem am 24. II. 1906 gehaltenen Vortrag bekannt geworden. An entsprechenden Stellen habe ich Bemerkungen aus dieser Arbeit nachträglich zugefügt.

Zeitschr. f. Hygiene. L.VI. 
d'Orient Napoleons mußte 1799 auf dem Feldzuge in Ägypten und Syrien die Belagerung von St. Jean d'Acre wegen der im Lager herrschenden Pest und Ruhr aufgeben. Im Jahre 1817 wurde ein englisches Heer in Italien durch die Cholera fast völlig vernichtet. Im Krimkriege endlich scheiterte die Belagerung von Sebastopol rom 9. X. 1854 bis Frühjahr 1855 an den furchtbaren Verheerungen, welche Krankheiten unter den französischen, englischen und türkischen Truppen anrichteten, und erst die im Frühjahr 1855 eintreffenden Verstärkungen ermöglichten eine Weiterführung des Krieges. In allen bisherigen Kriegen aber war die Zahl der in den Lazaretten behandelten Kranken bedeutend größer als die Zahl der dort behandelten Verwundeten. So wurden von 1000 Mann der Iststärke behandelt

1866 wegen Verwundung $49 \cdot 0$, wegen Krankheit $239 \cdot 3$

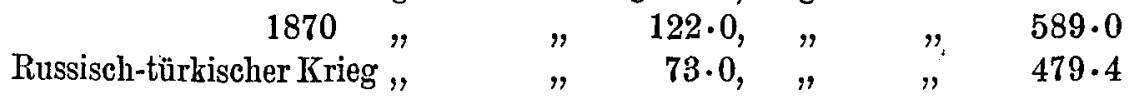

Im Burenkriege hatten die Engländer 22000 Verwundete, 450000 Kranke; fast die Hälfte aller Todesfälle war auf Typhus und Ruhr zurückzuführen.

Diese Krankheiten zu verhūten oder doch auf eine erheblich kleinere Zahl einzuschränken, daran nimmt einen bedeutenden Anteil die Versorgung mit gutem Trinkwasser; denn die wichtigsten Heeresseuchen Ruhr, Typhus, Cholera werden unter den Truppen durch das Trinkwasser verbreitet.

Dieses Ziel haben die Japaner im Kriege gegen RuBland nahezu vollkommen erreicht, da es ihnen gelang, den Zugang an ansteckenden Krankheiten auf 1.96 Prozent, den Verlust auf 0.76 Prozent herabzudrücken, ein Erfolg der vorzüglichen hygienischen Maßregeln im japanischen Heer, die General Oku mit den Worten anerkannte: „Die beste Waffe in unserem Feldzuge war nicht das Murata-Gewehr, sondern das Mikroskop." Der japanische Arzt war, so sagt die englische Zeitschrift Nature, mit Mikroskop und Chemikalien bei den vordersten Plänklern, um die Brunnen zu prüfen und mit Aufschriften zu versehen, damit das nachfolgende Heer kein verdorbenes Wasser trinke usw.

Die Wasservorräte der Natur, aus welchen wir unseren Bedarf an Trinkwasser zu decken imstande sind, treten uns in mannigfaltiger Weise, aber in ewig sich erneuerndem Kreislauf entgegen. Durch die Wirkung der Sonne rerdampft das Wasser der Ozeane und unzähliger kleiner Wasserfächen, welche die Erde bedecken; der sich kondensierende Wasserdampf kehrt als Tau, Nebel, Regen, Schnee, Hagel zur Erde zurück. Wo die Feuchtigkeit auf den Boden fällt, verdunstet ein Teil - ein Drittel 
etwa - sofort und kehrt in die Atmosphäre zurück; ein zweites Drittel läuft von der Erdoberfläche ab, um sich zu Bächen und Flüssen zu vereinen und im Strom zum Meer zurückzukehren, oder über undurchlässigen Schichten Pfützen, Tümpel, Teiche oder Sümpfe zu bilden. Das letzte Drittel sickert in den Boden und sinkt in diesem tiefer, bis es auf eine undurchlässige Schicht trifft, auf der es als Grundwasser verbleibt.

Man unterscheidet hiernach am besten Oberflächenwasser und unterirdisches oder Bodenwasser. Das Regenwasser nimmt auf seinem Wege durch die Atmosphäre aus derselben verschiedene Bestandteile auf; es ist deshalb nicht unter allen Umständen als ein reines und gesundes Wasser anzusehen. Zwar enthält es die kleinsten Mengen feuerbeständiger Stoffe, aber reichlich Staubteilchen und damit alle Mikroorganismen, welche sich eben im Staube der betreffenden Ortlichkeit zu finden pflegen. Noch melrr verunreinigt ist häufig Schneewasser, da der Schnee bei langem Liegen sehr bedeutende Mengen von Staub und Verunreinigungen aufzunehmen in der Lage ist. Auf der Erdoberfläche reiBt dann das Regenwasser Sand, Lehm, Schmutzteilchen und die diesen in groBer Zahl anhaftenden verschiedenartigsten Mikroorganismen an sich und führt sie in die nächsten Gewässer. Zu diesen Verunreinigungen kommen noch die vom Menschen herrührenden Verschmutzungen, als Haus- und Industrieabwässer, in denen sich viele Millionen von Keimen befinden. Alles Obertlächenwasser ist also mehr oder weniger der Gefahr der Infeltion ausgesetzt und zwar in um so höherem MaBe, je mehr sein ZufluBgebiet mit menschlichen Wohnungen besetzt ist, je mehr Schmutzwasser aus den menschlichen Ansiedlungen in dasselbe hineinlăuft, je näher die Infektionsstätten gelegen sind, je kleiner das aufzunehmende Gewïsser und je zahlreicher und stärker seine Zullüsse sind.

Das in den Boden sickernde Wasser tritt in verschiedenen Formen auf. Bald findet es sich fein verteilt überall in geringer Menge in den Poren der Gesteine, selbst der dichtesten, als Bergfeuchtigkeit oder BergschweiB, bald staut es sich über einer undurchlässigen Unterlage und füllt dann bis zu einer gewissen Höhe alle Poren und Klüfte als Grundwasser; bald flieBt es, gesammelt in einzelnen ausgewaschenen Gerinnen, als Quellader.

Auf seinem Wege von der Oberfläche bis zur undurchlässigen Schicht hat das Wasser oft meilenweite Strecken von gut filtrierendem Sande zu durchlaufen. Alle ihm beigemengten körperlichen Bestandteile, darunter auch die Mikroorganismen, werden hier vollständig oder größtenteils zurückgehalten. Die löslichen Bestandteile werden beim Durchtritt durch die Bodenschichten, soweit sie organische sind, je nach der Beschaffenheit and Dicke der Schicht in salpetrige oder Salpetersäure zerlegt. Die 
gelösten Salze gehen meist unverändert durch dicke Bodenschichten hindurch. Das Wasser gibt aber nicht nur an die durchflossenen Schichten ab; sondern nimmt auch aus denselben lösliche Stoffe auf; es wird also je nach der geologisehen Beschaffenheit des Bodens rerschiedene Beschaffenheit der in ihm gelösten Stoffe annehmen. Das Wasser ist deshalb vollkommen abhängig von dem umgebenden Wasserträger, und zwar ist die Menge der gelōsten Bestandteile um so größer, je längere Zeit Wasser und Gestein in Berührung miteinander waren oder, anders ausgedrückt, der Bestandteil an gelösten Stoffen ist bei gleicher Gesteinsart in denjenigen Wässern größer, welche sich in engen Poren bewegen, als bei solchen, welche weite Kanäle durchfließen; das Grundwasser bleibt nun je nach der Lage der undurehlässigen Schicht in einem Ruhe- und Gleichgewichtszustand stehen, bildet unterirdische Seen oder fließt nur ganz langsam, 1 bis höchstens $25 \mathrm{~m}$ in 24 Stunden als Grundwasserstrom weiter. Alle etwa noch in dem Grundwasser vorhandenen körperlichen Teile sinken deshalb vermöge ihres Schwergewichts zu Boden und sterben, soweit es Mikroorganismen sind, ab. Somit wird das Grundwasser keimfrei und bleibt es, solange es gegen Verunreinigung von oben her geschützt ist. Solches Grundwasser ist ein im hygienischen Sinne einwandfreies Trinkwasser.

Quellwasser ist unterirdisches Wasser, welches nach Durchsickern einer meist dünnen Erdschicht in Gesteinsspalten und Klüften, besonderen unterirdischen Kanälen und Rinnen einer meist nabeliegenden Ausflußoffnung in großer Schnelligkeit zueilt. Das Wasser hat weder Ruhe noch Zeit, die in ihm noch vorhandenen, schwebenden körperlichen Stoffe dürch Sedimentieren abzulagern. Es besitzt auch keine gleichmäBig bleibende Mlenge, sondern ist ganz und gar abhängig vom Regen. Bei heftigen Regengüssen findet man deshalb Quellen an Orten, wo bei trockner Jahreszeit kein Wasser aus der Erde hervorsprudelt; man findet aber auch unter denselben Umständen das sonst so klare Quellwasser trübe, als Zeichen der Verunreinigung durch nicht genügend filtriertes Oberflächenwasser.

Grundwasser ist alt, abgelagert, keimfrei, beständig in seiner Beschaffenheit; Quellwasser neu, von gestern, von heute, schnell wechselnd in seiner Beschaffenheit, stets der Gefahr der Verunreinigung ausgesetzt.

Wenn nun auch Grundwasser bei geeigneter Bodenschichtung zuweilen als Quelle zutage treten kann, so ist doch im Interesse der Verbraucher Quellwasser streng vom Grundwasser zu trennen. Eine große Zahl ausgedehnter Typhusepidemien hat sich zurückführen lassen anf Wasser, welches Quellen entnommen war. 
Wie soll nun gutes Trinkwasser beschaffen sein? Bekanntlich verlangt man, daB es klar, farb- und geruchlos, von erfrischendem Geschmack, frei von Krankheitskeimen und Giften sei.

Bei der Beurteilung des Trinkwassers haben wir also zunächst Farbe, Geschmack und Geruch zu prüfen. Das Wasser soll ohne auffallende Farbe und klar, also frei von sichtbaren Verunreinigungen und Trübungen sein. Eisenhaltiges Wasser zeigt oft gelbliche Färbung, Wasser aus Moorlagern und bituminösem Schiefer kann schwärzliche Furbe haben. Die Trübungen beruhen meist auf eingeschwemmten Erdteilchen, insbesondere Tonteilchen. Färbungen und Trübungen sind eigentlich nur Schönheitsfehler, sie machen das Wasser unappetitlich, aber nicht direkt gesundheitsschädlich. Das Eintreten von Trübungen in bis dahin klarem Wasser ist aber stets das Zeichen des Zuflusses von Oberflächenwasser. Die Trübungen deuten deshalb immer auf die Gefahr, daß mit ihnen auch Mikroorganismen Eingang in das Wasser gefunden haben können.

Der Geschmack hängt in erster linie von der Temperatur des Wassers ab. Erfrischend schmeckt Wasser nur, weun seine Temperatur nicht über $12^{\circ}$ hinausgeht. Wasser, welches fade, modrig, faulig, süBlich oder widerlich schmeckt, ist verunreinigt, meist durch Fäulnisstoffe, und kein Genußwasser.

Das Wasser soll keinen ausgesprochenen Geruch haben. Teerartiger Geruch weist auf Verbindungen mit Gasfabriken hin; Ammoniak- und Schwefelwasserstoffgeruch auf Zersetzungsvorgänge, auf rermodertes $\mathrm{Holz}$ in den Pumpen, abgestorbene Algen, Tierkadaver, Jauche- oder Abortgruben. Schwefelwasserstoff kann aber auch aus einer älteren Formation stammen; das Wasser kann dann nach Entfernung des Gases, durch Stehen an der Luft, vorzüglich sein. So enthalten die artesisehen Brunnen aus dem Tertiär in Ungarn, aus dem Diluvium in Böhmen meist reichlichen Schwefelwasserstoff.

Durch die sinnfälligen Veränderungen werden wir auf die Unappetitlichkeit und hierdurch auf die Unbrauchbarkeit als Trinkwasser aufmerksam gemacht. Weil nun diese Veränderungen oft auf Verschmutzung zurückzuführen sind und die Erfahrung gelehrt hat, da $B$ epidemische Krankheiten vorzugsweise da auftraten, wo Schmutzstoffe sich häufen; weil ferner das Brunnenwasser der meisten Städte auch dann, wenn es klar und wohlschmeckend ist, Schmutzstoffe, sogenannte Stadtlaugenstoffe, Fäulnisprodukte enthält, die durch die chemische Analyse als organische Stoffe, Ammoniak, salpetrige und Salpetersäure nachgewiesen werden können, wurde bei Beginn methodischer Wasseruntersuchungen die chemische Untersuchung maßgebend für die Wasserbeurteilung. Und als bei einigen größeren und kleineren Epidemien er- 
mittelt wurde, daB die ansteckenden Krankheiten durch Wasser vermittelt waren, in dem die chemische Untersuchung jene Schmutzstoffe in gröBerer Menge nachweisen konnte, hielt man die Ansicht für bewiesen, daß organische Stoffe, Ammoniak, salpetrige und Salpetersäure auf Fäulnisherde und auf Bakterien im Wasser hindeuten. Es wurde hierbei vorausgesetzt, daB jene chemischen Fäulnisprodulte auf demselben Wege ins Wasser gelangen wie die Bakterien, so daB da, wo die einen sich finden, auch für die anderen die Zngangswege offen sind. Es bedurfte also nur gewisser Grenzzahlen, um Normen für gutes Trinkwasser aufzustellen Die Utberschreitung dieser Grenzzahlen berechtigten den Chemiker dazu, Wasser als ungenieBbar, verdächtig, gesundheitsgefährlich zu bezeichnen. Erst als Fraenkel nachwies, dab die löslichen Stoffe nicht dieselben Wege einschlagen wie die Bakterien, daB sie auch da ins Grundwasser gelangen können, wo die Bakterien im Boden zurückgehalten werden, da erst erkannte man, daß die chemische Untersuchung keinerlei richtigen Hinweis auf die Infektionsgefahr eines Wassers zu geben vermag. Es blieb nunmehr nur übrig aus der Vermehrung der organischen Stoffe und ihrer Oxydationsprodulte den SchluB zu ziehen, daB Zersetzungsstoffe in das Wasser gelangen. Woher dieselben kommen, ob dieselben ihre Entstehung einer Zersetzung von Pflanzenresten früherer Erdperioden verdanken oder aus frischen Zersetzungsherden des menschlichen Haushaltes herrühren, darüber kann die chemische Untersuchung keine Auskunft geben, darüber kann nur die ortliche Besichtigung entscheiden. Aber auch für andere gelöste Stoffe, für Chlor, Kalk, Magnesia hatte man Grenzwerte festgesetzt. Wie schon erwähnt, hängt die Menge dieser Stoffe vollständig von den geologischen Verhältnissen ab und ist deshalb für die verschiedenen Orte sehr verschieden, überallgültige Grenzzahlen kann es also auch für diese Stoffe nicht geben; zur Abschätzung der Ergebnisse der chemischen Untersuchung brauchen wir somit einen MaBstab, mit welchem die gefundenen Resultate verglichen werden können. Ein solcher ist nur gegeben in den Vergleichszahlen, welche die Untersuchung reiner Wässer derselben Ortlichkeit ergibt. Jede Untersuchungsstelle, die Urteile über Wasserfragen abzugeben hat, muB sich deshalb für seine Interessensphäre einen solchen Maßstab schaffen. Eine Verunreinigung eines Wassers liegt nur dann vor, wenn nicht eine, sondern mehrere Zahlen über den gewöhnlichen, für den Ort maßgebenden Wert hinausschnellen. Solange im besonderen Chlor, Kalk, Magnesia nicht in Mengen im Wasser vorhanden sind, welche sich durch den Geschmack bemerkbar machen, ist ihre Menge in gesundheitlicher Beziehung ohne Belang. In Nietleben bei Halle haben wir z. B. ein sehr gut schmeckendes Tinkwasser, dessen Chlorgehalt zwischen 90 bis 100 mg Chlor im Liter beträgt, die 
höchste Grenzzahl für Chlor war $\mathbf{3 3}{ }^{\mathrm{mg}}$. Kalk und Magnesia bedingen die Härte des Wassers, sie machen es unbequem für die Hausfrau, finden sich aber niemals in solchen Mengen, daß sie die Gesundheit schädigen. Man muB es als eine Mär bezeichnen, daB hartes Wasser die Gesundheit schädigt; es gibt nicht bloB einzelne Häuser, sondern ganze Ortschaften, in denen Wasser mit 100 Härtegraden ohne Nachteil getrunken wird. Auf Grund der chemischen Analyse einer Wasserprobe läBt sich demnach ein Urteil über die hygienische Beschaffenheit, besonders die Infektionsgefahr des Wassers, nicht fällen. Wir sind bei der Verwertung ihrer Ergebnisse außerdem auf einen Maßstab, den wir im Kriege nicht besitzen, und auf die örtliche Untersuchung angewiesen. Wir werden deshalb im Felde auf sie verzichten und sie durch die Ortsbesichtigung ersetzen können. Im Frieden bei Neuanlagen von Wasserentnahmestellen können wir dieselbe nicht entbehren, weil Industrie und Haushalt hohes Interesse an der Härte des Wassers haben. Auch sind wir bei der Feststellung von Giften auf sie angewiesen, welche im Kriege bei der Feldarmee kaum in Frage kommen.

Bietet nun die bakteriologische Untersuchung sicherere Ergebnisse für die Trinkwasserbeurteilung?

Unter Umständen kann die bakteriologische Untersuchung anßerordentlich viel leisten. Finden wir pathogene Keime im Wasser, so ist es ganz bestimmt zu rerwerfen. Wie selten gelingt dies aber. Sind doch jetzt noch die Fälle zu zählen, wo im Wasser, das zweifelsohne die Veranlassung ausgedehnter Typhusepidemien abgab, der Typhuserreger nachgewiesen werden konnte. Man hat deshalb die Arten und die Zahl nicht pathogener Keime als Kennzeichen zur Beurteilung des Wassers herangezogen. Gewisse Arten können unter Umständen auf eine Verseuchung des Wassers hindeuten. So kann der Befund großer Mengen von Bact. coli im Wasser ein Hinweis auf Beimengung von Fäkalien sein. Außer in der Stuhlentleerung von Menschen kommt Bact. coli aber auch in dem Kot der Tiere vor; es ist ferner befähigt auch auBerhalb des Darmes zu leben; man darf es im Bereich gedüngter Erde überall erwarten, und man findet es auch in den besten, bakterienhaltigen Wässern, wenn man nur genügende Mengen untersucht. Man kann dem Bact. coli eine besondere Bedeutnng deshalb nur dann beilegen, wenn durch genaue Besichtigung der örtlichen Verhältnisse und der Filtrationskraft des Bodens die Infektionsmöglichkeit nachgewiesen wird. Die Zahl der Bakterien hat einen großen Wert als Antwort auf die Frage: Wie filtriert der Boden? Ist das Wasser bakterienfrei, so ist die Filtration ausreichend, es sind eben keine Wege vorhanden, auf denen überhaupt Organismen in dasselbe ein- 
dringen können. Aber auch in diesem Falle ist aus der Untersuchung einer einzelnen Probe kein SchluB zu ziehen. Es können gefährliche Fehler vorhanden sein, die nur augenblicklich keine Bakterien zuführen, z. B. infolge Trockenheit der Zuflüsse. Erst fortgesetzte Untersuchungen mit demselben Ergebnis können hier Sicherheit gewāhren. Sind aber ziemlich viel oder sehr viele Bakterien nachgewiesen, dann brauchen diese durchaus nicht auf Infektionsgefahr zu deuten. Wir wissen z. B., daB bei Brunnen die Menge der Keime abhängig ist von so verschiedenen Dingen, von der Vermehrungsfähigkeit im Wasser, Temperatur, Tiefe, Benutzung des Brunnens, Grundwassermenge, schlieBlich auch von der Art der Entnahme des Wassers, daB auch eine hohe Zahl saprophytischer Keime kein sicheres Urteil darüber ermöglicht, ob Einfuhrwege für eine Infeltion vorhanden sind. Auch hier bleibt die Entscheidung über die Tauglichkeit des Wassers vollkommen abhängig von dem Ergebnis der örtlichen Besichtigung. Ich will nicht noch auf die Schwierigkeiten und die Zeitdauer eingehender bakteriologischer Wasseruntersuchungen eingehen. Soviel ist sicher, daß man die Beurteilung des Trinkwassers bei der Feldarmee nicht von einer bakteriologischen Untersuchung abhängig machen kann. Wir können sie auch ganz gat entbehren, da die örtliche Untersuchung in den weitaus meisten Fällen ganz allein ausschlaggebend für die hygienische Beurteilung des Wassers ist. Was wollen wir denn beim Trinkwasser vorzngsweise untersuchen? Ob Infeltionsgefahr vorhanden ist. Nun ist diese eben nur vorhanden, wenn grobe Wege vorhanden sind, auf welchen Mikroorganismen ins Wasser gelangen können. Ergibt die Besichtigung, daB ein offenes Wasser vorliegt, so ist damit die Gefahr der Infektion gegeben: sie ist naheliegend, wenn die Umgebung der Wasserbezugsquelle oder ihr ZufluBgebiet dem menschlichen Verkehr ausgesetzt oder leicht zugänglich ist, sie ist fernliegend, wenu der Träger der Keime, der Mensch, dem Wasser fern bleibt. Ein. Bach, welcher aus dem von Menschen selten betretenen Hochwald hervortritt, ein Stauweiher, welcher im engen waldigen Gebirgstal seine Existenz verbirgt, eine Quelle, welche ihr Wasser von den Hängen dichtbewaldeter Hügel bezieht oder am Rande einer ungedüngten Bergwiese hervorbricht, ein offener Brunnen, der abseits vom Gehöfte mitten in dem ungedüngten Obstgarten eines Gutes liegt, sind, obgleich sie offene Wässer darstellen, doch der Gefahr einer Infektion in einem so geringen Grade ausgesetzt, daß diese gar nicht in Betracht kommt. Wollte man auch bei solchen Wässern mit Infektionen rechnen, weil sie Oberflächenwässer sind, so gäbe es außer in den tiefstehenden Schichten des Grundwassers überhaupt kein infektionssicheres Wasser. Selbst die Mehrzahl der Quellen ist der Infektion viel mehr ausgesetzt als die eben erwähnten Wässer. 
Bei den nicht offenen, den unterirdischen Wässern, ist die Infektionsgefahr geringer, aber nicht ganz ausgeschlossen. Die Möglichkeit der Infektionsgefahr liegt dann vor, wenn eine nicht genügend filtrierende Erdschicht vorhanden ist oder wenn die Filtration ganz fehlt, wenn Oberflächenwasser durch Spalten und Klüfte oder groben Schotter rasch in den Boden versinkt und bald zum Genuß kommt.

Bei der Beurteilung von Grundwasserbrunnen haben wir also vor allen Dingen zu prüfen, ob die Lage und Art des Brunnens und seine Bedeckung. das Eindringen von Schmutzwasser ausschlieBt. Zunächst untersuchen wir, falls die Lage nicht schon die Infektion unwahrscheinlich macht, ob oberflächliche Einflüsse durch Spalte und Risse in und unter der Deckung möglich sind; sodann ob der Brunnenkessel durchlässig ist. Man läBt zu diesem Zweck den Brunnendeckel abnehmen und prüft die Wandung auf Streifen und Flecke; man kann durch Ausgießen gröBerer, eventuell gefärbter Wassermengen die Einläufe deutlicher sichtbar machen. Sind die oberflächlichen Zuflüsse genau untersucht, dann fragt es sich noch, ob unterirdische Zuflüsse bestehen, die Mikroorganismen zuführen können. $\mathrm{Da}$ ist die Bodenbeschaffenheit und der Tiefstand des Grundwasserstandes zu betrachten. Besichtigung in der Nähe gelegener Gruben oder Aufgraben des benachbarten Bodens in 1 bis $1 \frac{1}{2} \mathrm{~m}$ Tiefe geben einen AufschluB, ob ein Durchtritt von Oberflächenwasser mit Mikroorganismen durch den gewachsenen Boden möglich, oder ob die Filtration eine gute ist. Endlich ist noch auf künstliche Risse oder Gänge zu achten, welche den Brunnen mit Senkgruben, Schlammfängen verbinden können.

Die Appetitlichkeit wird gleichfalls in erheblichem Maße durch die Ortlichkeit beeinflußt. Ergibt die Besichtigung, daß dicht neben den Aborten und Dungstätten, welche wohl von ihren gelösten, aber wegen der guten Bodenfiltration nichts von ihren körperlichen Bestandteilen abgeben, sich ein Trinkbrunnen befindet, so wird der Geschmack des normalen Menschen das Wasser als nicht appetitlich zurückweisen. Dasselbe wird eintreten, wenn dicht über der Quellmündung an den Papierresten erkannt werden kann, daB ein Tonnenwagen dort Abtrittsjauche über die Wiese zwecks Düngung verstreut hat. FlieBt ein Bach durch einen Ort, so erscheint uns sein Wasser unterhalb unappetitlich, weil wir wissen, daß allermöglicher Unrat hineingeworfen wird, ein Punkt der bei Anlage ron Biwaks besonders zu beachten ist, weil die Felddienstordnung der Wasserversorgung halber die Nāhe von Ortschaften für Biwakplätze ausdrücklich hervorhebt. Bei Bächen und Flüssen ist die Art der verunreinigenden Zuflüsse festzustellen; ob Abwässer aus menschlichen Wohnungen, Abflüsse von den angrenzenden gedüngten Feldern hinein- 
gehen, ob Fischer auf dem Flusse leben, ob städtische oder Industriewässer hineingehen. Charakteristisch für letztere sind besonders die flottierenden Rasen des Sphaerotilus natans und die schafwolleähnlichen am Grunde des Gewässers an Steinen und Zweigen haftenden Flocken der Beggiatoa.

Die örtliche Besichtigung gibt uns also schnelle Auskunft auf die wichtigste Frage bei Beurteilung des Trinkwassers, nämlich ob eine Infektionsgefahr vorhanden ist. Im Felde wird deshalb der in der Wasserbeurteilung geübte Sanitätsoffizier auf Grund der örtlichen Besichtigung und der grobsinnlichen Prüfung in fast allen Fällen ein zutreffendes Urteil über die Brauchbarkeit des Trinkwassers abgeben können.

Gutes Trinkwasser finden wir unter den gegebenen Voraussetzungen im Grundwasser. Dieses wird durch Brunnen erschlossen. Es wird deshalb, bei der Versorgung der Truppen mit gutem Trinkwasser, unser Augenmerk notwendigerweise vor allen Dingen auf Auswahl guter Brunnen zu richten sein, deren Schutz vor Verunreinigungen den benutzenden Truppen zur Pflicht zu machen. ist. Wenn man solehe Brunnen nicht findet, wird man darnach streben, durch Neuanlage von Brunnen Grundwasser zu erschließen. Zuerst haben die Engländer bei ihrer Expedition nach Abessinien von diesem Verfahren Gebrauch gemacht. Sie benutzten hierzu den von einem Amerikaner erfundenen, bei uns als abessinischer Brunnen bekannten Apparat. Dieser besteht aus dem eisernen Pumpenständer mit Schwengel, Kolbenstange und Pumpenkolben; aus einem zerlegbaren, durch Verschraubung der einzelnen Teile beliebig zu verlängernden Saugrohr, endlich aus dem Filterrohr mit der massiven Rammspitze. Mit Hilfe eines eisernen Fallblocks wird zuerst das unterste Stück in die Erde eingerammt; auf dieses wird Stück für Stück das Saugrohr angeschraubt und eingerammt, bis die wasserführende Schicht erreicht ist. Das erste ausgepumpte Wasser ist stets trübe, erst bei fortgesetztem Pumpen wird das Wasser klar. Die Aufstellung erfolgt von geübten Arbeitern in 2 bis 3 Stunden, die Abrüstung beim Weitermarsch der Truppen in der halben Zeit. Bei Auswahl geeigneter Ortlichkeiten liefern diese Brunnen ein gutes, von schädlichen Verunreinigungen freies Wasser. Sowohl bei der Expedition nach China wie in Afrika waren die Truppen - Bataillon, Abteilung, Kavallerie-Regiment - mit solchen Brunnen ausgerüstet. Leider gelang es wegen des zu geringen und schlechten oder gänzlich fehlenden Grundwassers in der Peihoebene sowie im felsigen Gelände Afrikas nicht, einen erfolgreichen Gebrauch von den Brunnen zu machen. Um so erfreulicher und lehrreicher ist deshalb ein bei den letzten Herbstübungen auf Veranlassung des Herrn Generaloberarztes Schmiedicke ausgeführter Versuch, Truppenteile der 8. Division im 
Biwak aus Abessinierbrunnen mit Wasser zu versorgen. Es gelang hierbei, eine Brunnenanlage zu schaffen, welche den Wasserbedarf einer Division gedeckt hätte. Die Vorteile einer guten Wasserversorgung der Truppen sind so groB, daB derartige Improvisationen zu Aufgaben für Sanitätsoffiziere und Pioniere bei Friedensübungnn zu machen sich im Ernstfall sicher belohnen würde.

Sind Truppen auf Oberflächenwasser angewiesen, so wird in den meisten Fällen eine Reinigung und Sterilisierung erforderlich werden. Die Sehädlichkeiten, welche dem Wasser beigemengt sein können, sind teils chemisch gelöst, teils mechanisch suspendiert. Die chemisch gelösten Stoffe sind, solange wir das Wasser überhaupt zu trinken vermögen und sie nicht Gifte sind, nicht gesundheitsgefährlich; sie kommen für die Wasserreinigung im Felde nicht in Betracht. Die suspendierten Verunreinigungen sind teils gröberer, teils feinerer Art. Die gröberen bestehen in der Regel aus Sand, Pflanzenteilen, Algen und dergleichen; sie trüben das Wasser und sind durch grobe Filter (Leinewand, FlieBpapier, Haarsiebe, reine Beutel und ähnliches) leicht zu entfernen. Wichtiger und ungleich schwieriger zu beseitigen sind die feineren Verunreinigungen des Wassers, welche keine Trübungen machen und nur bei mikroskopischer Betrachtung erkannt werden. Teils sind es Infusorientierchen verschiedener Art, welche sich regelmäßig finden, wenn verwesende Pflanzenteilchen im Wasser vorhanden sind; teils sind es niedere pflanzliche Organismen. Die völlige Vermichtung dieser Organismen gehört zu den schwierigsten Aufgaben der hygienischen Technik. Man hat diese Aufgabe auf verschiedenen Wegen zu lösen versucht:

1. Durch Zusatz von Chemikalien.
2. " Filtration.
3. " Siedehitze.

Bei der Sterilisation des Wassers auf chemischem Wege sollen die angewandten Chemikalien entweder eine mechanische Ausfällung der suspendierten Stoffe, auch der Bakterien, bewirken, oder sie sollen die Bakterien direkt vernichten. Alle diejenigen Mittel, welche die Reinigung des Wassers nur durch eine mechanische Ausfällung bewirken, wie Kalkwasser, Alaun, Eisensalze, Kalipermanganat, erzeugen einen mehr oder weniger groben, flockigen Niedersehlag, weleher wohl die suspendierten Teile und auch einen groBen Teil der Bakterien mit zu Boden reibt, das Wasser nach verhältnismäBig kurzer Zeit zwar klärt, aber nicht keimfrei macht. In bezug auf die klärende Wirkung sind Liq. ferr. sesquichl. und Natr. bicarb. einerseits, Kalkwasser und Alaun anderseits annähernd gleichwertig. Wegen der geringen Beeinträchtigung des Geschmacks ist 
aber das Verfahren mit Eisenchlorid vorzuziehen. Von diesem Verfahren ist sowohl in China wie in Afrika von unseren Truppen in groBem Umfange Gebrauch gemacht worden, und es wird auch in der Zukunft für die Wasserversorgung im Felde nicht gänzlich entbehrt werden können. Zur Vernichtung der Keime im Wasser ist eine überaus große Zahl von Chemikalien rersucht und empfohlen worden. Schumburg hat etwa 20 verschiedene Verfahren einer Prüfung unterzogen und nachgewiesen, daß sie sämtlich nicht geeignet sind, dem Soldaten ein einwandfreies Wasser im Kriege zugänglich zu machen. Einzelne lassen gar keine, andere nur eine geringe Wirksamkeit erkennen; wieder andere brauchen sehr lange Zeit zur Entfaltung ihrer Wirksamkeit, noch andere machen durch die großen benötigten Mengen das Wasser zum Genuß unbrauchbar. Eine besondere Erwähnung verdienen nur Chlor, Brom und Ozon.

Chlor wurde in Form von Chlorkalk zuerst von Traube, sodann als Natriumhypochlorit (Eau de Labarraque) von Hünermann, Brom in Form einer Brom-Bromkalilösung von Schumburg zur Wassersterilisation verwendet. Chlor wird nach Entfaltung seiner Wirksamkeit durch Natriumsulfit, Brom durch Ammoniak aus dem Wasser entfernt. Nach 5 bis 10 Minuten langer Einwirkung sollten Cholera- und Typhuskeime sicher abgetötet sein. Schüder hat aber den Nachweis geliefert, daß Chlor wie Brom die pathogenen Krankheitskeime nicht immer im Wasser abzutöten vermögen, wenn sie auch unzweifelhaft die Fähigkeit besitzen, die Zahl derselben zu vermindern. $\mathrm{Da}$ es nun nicht gleichgültig ist, in welcher Menge und inwieweit in ihrer Entwicklungsfähigkeit geschädigte Krankheitskeime vom Menschen aufgenommen werden, so wird man, wenn kein besseres Mittel zur Verfügung steht, das Schumburg'sche oder Hünermann'sche Verfahren anwenden können. Zum Gebrauch im Notfall wurden unsere Truppen bei den überseeischen Expeditionen mit den zum Schumburg'schen Verfahren notwendigen Chemikalien und Geräten ausgerüstet.

Das Ozon ist ein Gas von knoblauchähnlichem Geruch, welches sich in geringer. Menge im Wasser löst, in diesen Lösungen abtötend auf die Keime wirkt und dabei gleichzeitig einen Teil der organischen Substanzen unter Entfärbung des Wassers und Verbesserung des Geschmacks verbrennt. Nach seiner chemischen Zusammensetzung ist das Ozon von dem Sauerstoff dadurch verschieden, daB sein Molekül nicht aus zwei, sondern aus drei Atomen besteht. In oxydierbaren Körpern geht von den drei Atomen eins in Reaktion, und es wird aus dem Ozon wieder der zweiatomige Sauerstoff, so daB also nach Wirkung des Ozons im Wasser nur gewöhnlicher Sauerstoff zurückbleibt und zwar etwa soviel als beispielsweise in dem für Enteisenungszwecke gelüfteten Wasser enthalten ist. 
Was die Herstellung anbetrifft, so gibt es einen chemischen und einen elektrischen Weg. Für seine Herstellung in der Praxis kommt nur der Weg über die elektrische Hochspannung in Frage, der darin besteht (Fig. 1), daB gewöhnliche Iuft in Ozonapparaten der Einwirkung der stillen blauen Entladung ausgesetzt wird. Die Luft wird behufs besserer Ozonausbeute durch Chlorcalcium vorgetrocknet, mittelst Gebläses unter schwachem Druck durch den gleichmäBig blau leuchtenden Entladungsraum des Ozonapparates mit solcher Geschwindigkeit getrieben, daB sie mit einem für die vorzunehmende Sterilisation geeigneten Prozentsatz von Ozon den Apparat verläßt. Die Menge des anzuwendenden Ozons muB für jedes Wasser wegen der verschiedenen Menge organischer Substanzen festgestellt

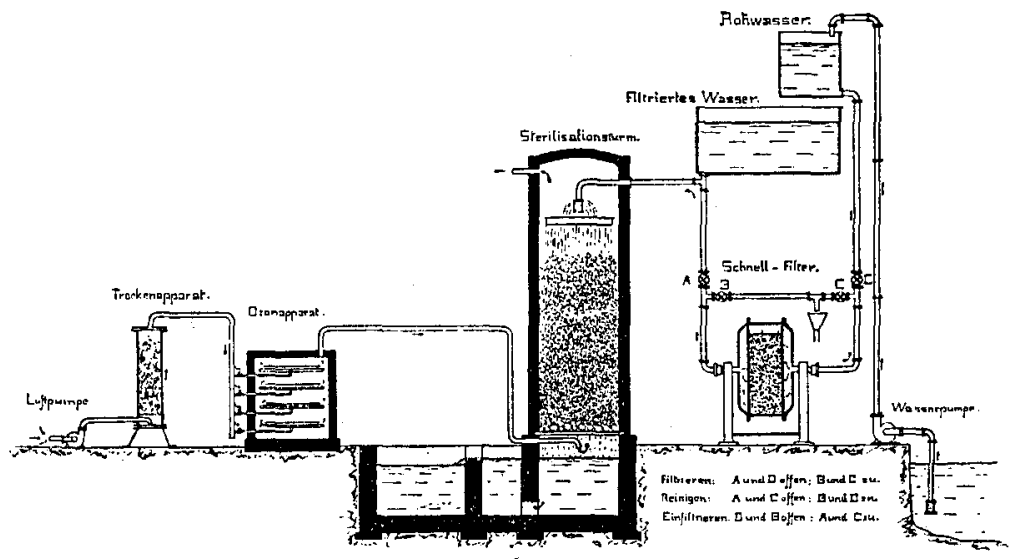

Fig. 1.

Schematische Darstellung des Martinikenfelder Versuchs-Ozonwasserwerks für Oberfläehenwasser mit Schnellfilteranlage zur mechanischen Vorreinigung des Rohwassers.

werden, was mit Hilfe von Jodkali-Lackmuspapier leicht möglich ist. Das so erhaltene 0zon wird in dem Sterilisationsturm mit dem durch eine Brause fein verteilten und durch Kiespackung laufenden Wasser in Berührung gebracht und darin teilweise in Lösung übergeführt, so daß es seine keimtötende Wirkung entfalten kann. Die ausführlichen Untersuchungen von Ohlmüller sowie diejenigen von Proskauer und Schüder haben bewiesen, daß durch die Ozonisierung eine so vollkommene Abtötung der im Wasser enthaltenen pathogenen Keime erfolgt, daß auch durch das Anreicherungsverfahren solche nicht mehr nachgewiesen werden konnten. Das Ozon ist also ein durchaus sicheres Wassersterilisationsmittel, welches außerdem den Vorteil hat, daB das gereinigte Wasser an Farbe und Geschmack nicht nur nicht beeinträchtigt, sondern verbessert wird. Die 
Firma Siemens \& Halske, welche sich bei uns in Deutschland um die Vervollkommnung dieses Verfahrens besonders verdient gemacht hat, hat auch eine fahrbare Ozonanlage (Fig. 2) gebaut, die zu Vorversuchen bei in Aussicht genommenen Ozonanlagen im GroBen benutzt wird. Sie besteht aus einem Petroleum-Antriebsmotor und einem Wagen mit vollständiger Sterilisationseinrichtung. Für den Gebrauch im Felde geeignete fahrbare Ozonsterilisationsanlagen wurden von der russischen Armee auf dem mandschurischen Kriegsschauplatz verwendet. Eine solche Anlage setzt sich zusammen aus 2 Wagen, einem Maschinen- und einem Sterilisationswagen. Die Skizze (Fig. 3) stellt links den Inhalt des Maschinen-, rechts den des Sterilisationswagens dar. Auf dem Maschinenwagen sind untergebracht: 1. Ein Benzinmotor von der Konstruktion der Automobil-

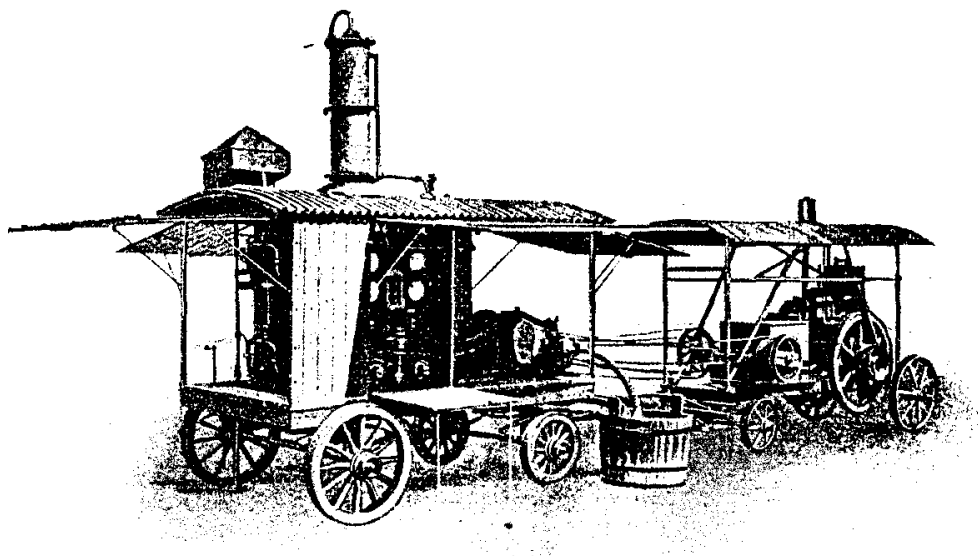

Fig. 2.

Transportable Siemenssche Ozonanlage für $3^{\mathrm{cbm}}$ Stundenleistung.

motoren, 2. eine mit dessen Achse direlst gekuppelte Wechselstrommaschine mit Gleichstromerregerdynamo zur Erzeugung des niedrig gespannten primären Wechselstroms für den Transformator, 3. eine kleine Zahnradpumpe, welche das Rohwasser ansaugt und in die Apparate des Sterilisationsturmes drückt, 4. ein kleines Gebläse, welches die Luft, durch Chlorcalcium im Turm schwach vorgetrocknet, für den Ozonapparat und den Turm des Sterilisationswagens liefert, 5. Kasten mit Reserveozonröhren und Reserveteilen für den Benzinmotor. Der Sterilisationswagen enthält: 1. Zwei federnd gelagerte Siemens'sche Ozonkasten mit 8 Ozonröhrenelementen, 2. einen unter den Ozonapparaten stehenden Transformator zur Erzeugung von Hochspannung aus den vom Maschinenwagen durch Kabelverbindung kommenden, niedrig gespannten primären Wechsel- 
strömen, 3. drei parallel gehaltene Schnell- oder Vorfilter, bestehend aus Blechzylindern, in welchen Filtersäcke hängen, durch die das Rohwasser vor Eintritt in den Turm gedrückt und dabei. von gröberen Schwebestoffen befreit wird, 4 . einen $2,5^{\mathrm{m}}$ hohen, $0,2^{\mathrm{qm}^{\mathrm{m}}}$ weiten mit Verteilungsmaterial (taubeneigroßem Kies oder zementüberzogenem Bimstein) versehenen runden Sterilisationsturm aus Eisenblech, der aus zwei auf einander gesetzten Teilen mit je einem durchlöcherten Boden besteht, und der nach einer Seite umlegbar angeordnet ist, 5. Kasten mit Werkzeug und Reserveteilen.

Beim Betriebe stehen die Wagen (im Sinne der Fig. 4 und 5) nebeneinander. Durch den auf der Abbildung sichtbaren dicken Sang und Druck-

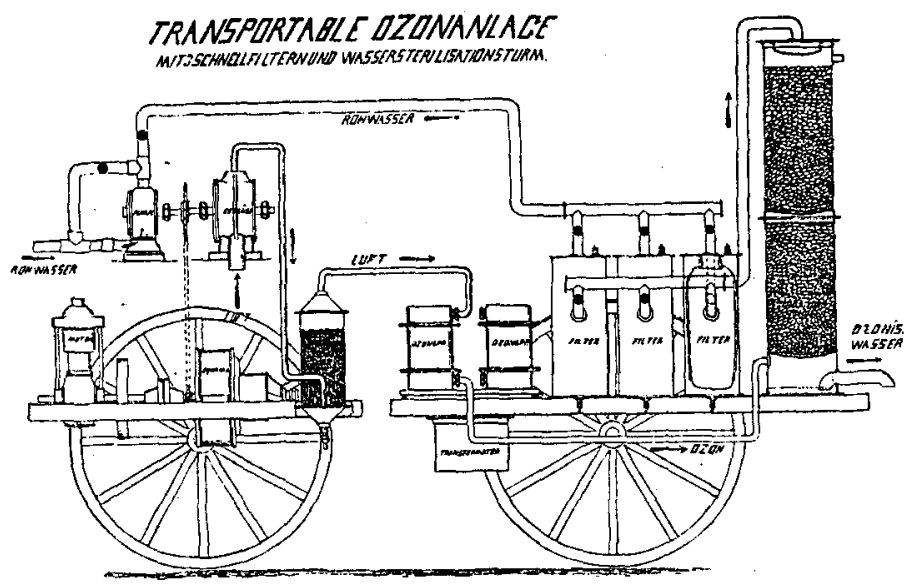

Fig. 3.

schlauch wird das Rohwasser von der Wasserpumpe des Maschinenwagens aus in die Schnellfilter und den Sterilisationsturm gebracht. Durch den daneben liegenden dünneren Luftsauge- und Druckschlauch geht die Luft vom Gebläse des Maschinenwagens in den Ozonapparat und von hier aus in den unteren Teil des Sterilisationsturmes, während durch das ebenfalls sichtbare Kabel der primäre Strom der Wechselstrommaschine des Maschinenwagens in den Transformator des Sterilisationswagens geleitet wird. Während der Fahrt (Fig. 6) liegt der umgelegte Turm in zwei Teilen auf dem Sterilisationswagen, die drei Schnellfilter dagegen auf dem Maschinenwagen. Jeder Wagen ist für Bespannung mit einem Pferde eingerichtet und hat ein Gewicht von $90 \mathrm{~kg}^{\mathrm{kg}}$. Die Anlage ist für eine Leistung von 2 bis $3^{\text {cbm }}$ Wasser in der Stunde eingerichtet. 
Utber die mit dieser fahrbahren Ozonsterilisationsanlage gemachten Erfahrungen ist ein Urteil nicht veröffentlicht worden; Oberstabsarzt Schaefer hat sie, wie er mir mitteilte, nicht zu sehen bekommen ${ }^{1}$. Die Prüfung der bakterienabtötenden Leistungsfähigkeit der Apparate hat zu sehr guten Ergebnissen geführt. Hat der Apparat den Anforderungen im Felde gleich gut entsprochen, so wäre ein weiterer bedeutender Fortschritt in der Beschaffung guten Trinkwassers erreicht.

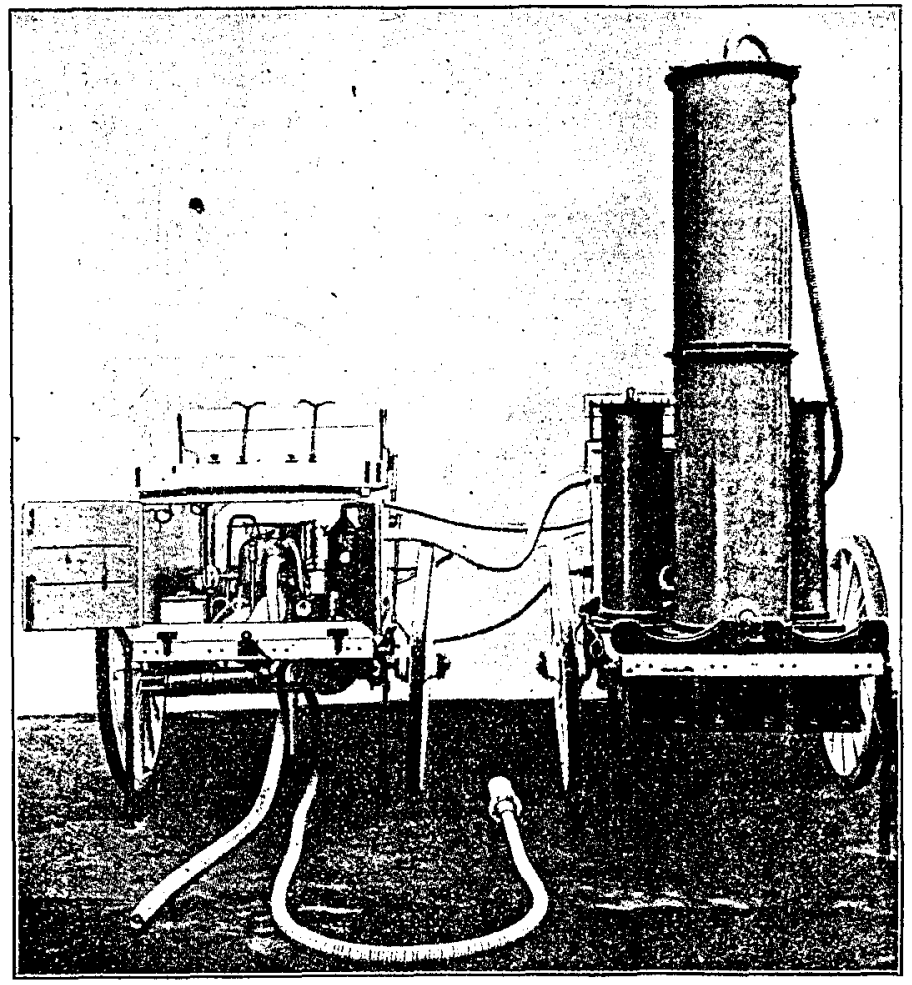

Fig. 4.

Der zweite Weg, auf dem eine Wasserreinigung erzielt wird, ist der der Filtration.

Für die Wasserfiltration im Großen ist mit Erfolg die Sandfiltration des Erdbodens nachgeahmt worden. Im Felde wäre es gewiß das Einfachste, die Filtrationskraft des Erdbodens selbst auszunützen. Die Kriegs-

${ }^{1}$ Nach Mitteilung von Hrn. Generaloberarzt Dr. Musehold sind diese Apparate den Russen von den Japanern abgenommen worden, ehe sie in Betrieb gesetzt wurden. 
Sanitäts-Ordnung empfiellt deshalb die Herstellung einfacher Filtertonnen, auf deren Boden eine dichte Schicht von Kies, kleiner Steine, aschefreier Holzkohle, kurzes Stroh, reine Wolle getan wird. Derartige Tonnen solle man in der Nähe des Ufers von größeren Gewässern eingraben und alsdann eine kleine zweite Tonne hineinstellen. Selten wird man aber Verhältnisse finden, wo bei hinreichender Wassermenge eine gut filtrierende Erdschicht in der Nähe groBer Gewässer vorhanden ist. Die in die Tonne eingebrachten Materialien balten natürlich nur die groben Verunreinigungen zurück. Für die Filtration im kleinen hat man eine grobe Zahl von

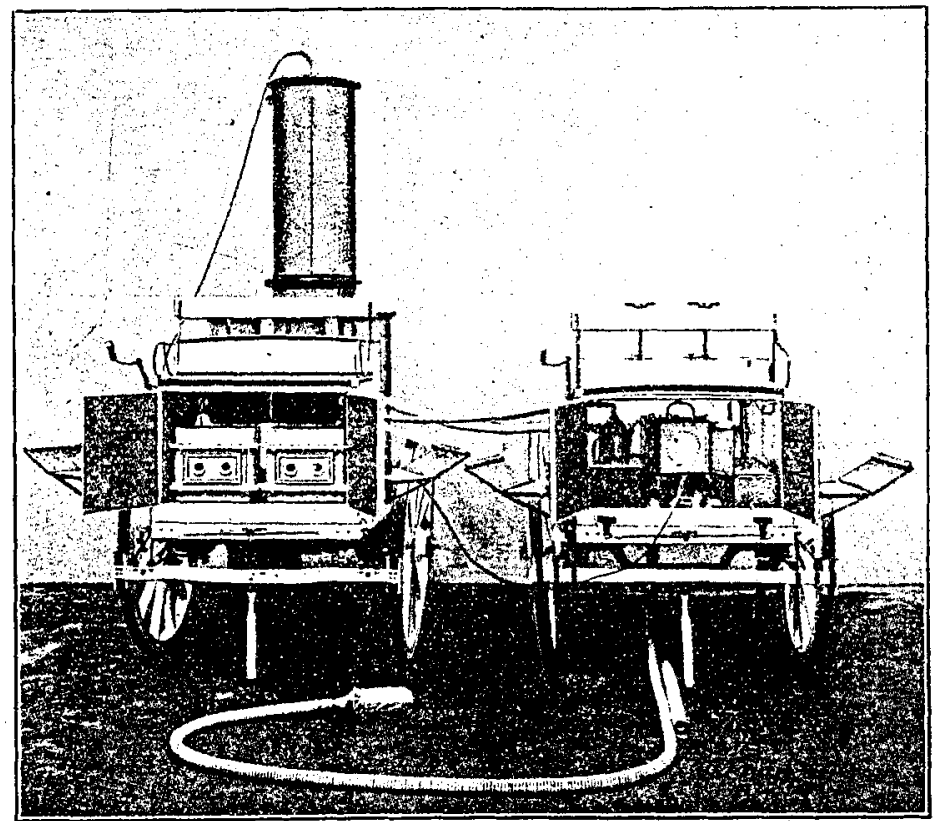

Fig. 5.

Apparaten ersonnen, welche aus dem verschiedensten Material hergestellt sind. Am bekanntesten sind geworden die Filter aus Koble, Eisenschwamm, Filz, Frieswolle, Zellulose, Sandstein, Bimsstein, Papier, Tierkohle, porösem Ton, Asbest, Porzellanerde, Kieselgur. Alle diese Filter haben sich mehr oder weniger unbrauchbar, ja sogar schädlich erwiesen insofern, als sie nicht nur nicht die Krankheitskeime zurückzuhalten vermögen, sondern geradezu zu Brutstätten für solche wurden, so daß der Keimgehalt des filtrierten Wassers den des unfiltrierten oft um das Hundertbis Tausendfache übertrifft. Eine genügende Bakterien-zurückhaltende Zeitschr. f. Hygiene. LVT. 
Wirksamkeit besitzen nur die Filter aus Asbest, Porzellanerde und Kieselgur und dies auch nur für die ersten Tage ihrer Tätigkeit, denn die anfangs zurückgehaltenen Keime durchwachsen in einigen Tagen, namentlich in der Wärme, das ganze Filter. Es ist deshalb eine strenge Uberwachung durch bakteriologische Wasseruntersuchung und häufiges Sterilisieren der Filter unbedingtes Erfordernis bei ihrer Verwendung. Aus Asbest lassen sich für Wasser leicht durchgängige, võllig keimdichte Filterscheiben herstellen. Diese vertragen aber das Kochen nicht und auch durch desinfizierende Flüssigkeit wird vielfach die Kittsubstanz gelockert und aufgelōst. Für die Truppen sind sie schon ihrer geringen Haltbarkeit wegen nicht verwendbar.

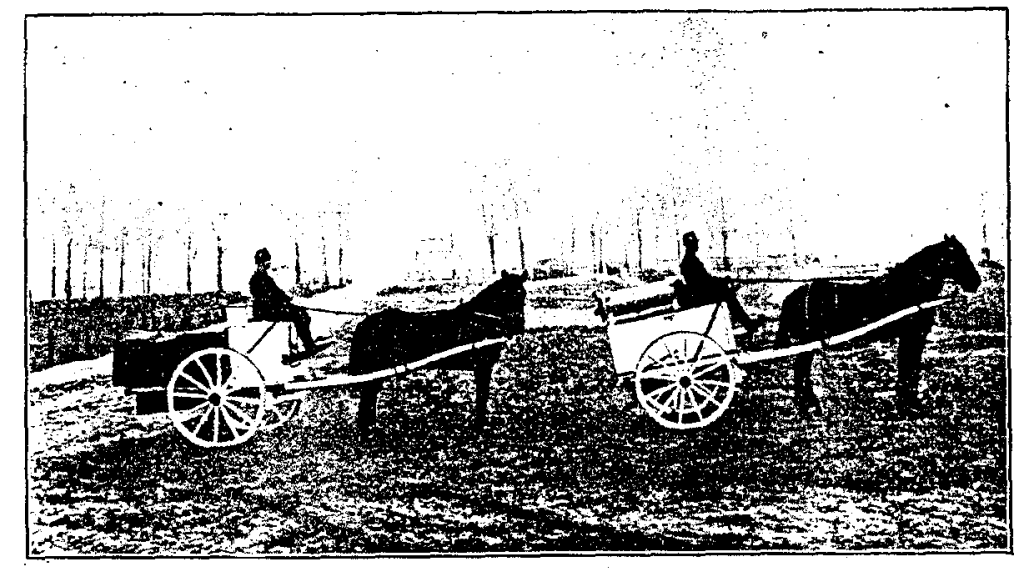

Fig. 6.

Militär-Ozon-Wagen (Kraft- und Sterilisationswagen der Figuren 4 nnd 5) auf der Fahrt.

Die Filter aus Porzellan, die sogenannten Pasteur-Chamberlandschen Filter, welche in der französischen Armee eingeführt sind, entsprechen allen Anforderungen an Keimdichtigkeit, auch ihr Material ist ein recht brauchbares. Ihre praktische Verwendbarkeit wird aber durch gar zu geringe Ergiebigkeit sehr beeinträchtigt, und auch die Dauer ihrer Keimdichtigkeit ist eine sehr begrenzte.

In Deutschland liefert die Berkefeldfilter-Gesellschaft sehr brauehbare Filter. Sie bestehen bekanntlich aus einem Zylinder ausgebrannter Diatomeenerde, dem Kieselgur; dieser ist in einer Metallhülse befestigt. Das Wasser tritt von unten her zwischen Metallhülle und Kerze, filtriert durch diese hindurch in das Innere der Kerze hinein und verläBt sie 
durch ein Ansatzrohr. Die Reinigung und Sterilisierung der Kerze muB bei fortgesetztem Gebrauch tăglich ausgeführt werden; sie erfolgt durch Abbürsten mit Loofah und Auskochen in 2 Proz. Sodalösung. Letzteres namentlich muB sehr vorsichtig ausgeführt werden wegen der großen Zerbrechlichkeit der Kerzen, die hierbei sehr leicht Sprünge bekommen. Der zum Hindurchtreiben des Wassers durch die Kerze erforderliche Druck wird bei dem Armeefilter durch eine Druckpumpe erzeugt, welche mit dem Kerzenmantel in Verbindung gebracht ist und deren Stöße durch einen eingeführten Windkessel ausgeglichen wird. Die BerkefeldfilterGesellschaft hat verschiedene Modelle und Größen von Armeefiltern konstruiert.

Der kleine tragbare Armeefilter $\mathrm{M} / 1905$ (Fig. 7), weleher versuchsweise bei verschiedenen Truppen im letzten Manöver mitgeführt wurde, kann, in eine Segeltuchtasche verpackt, von einem
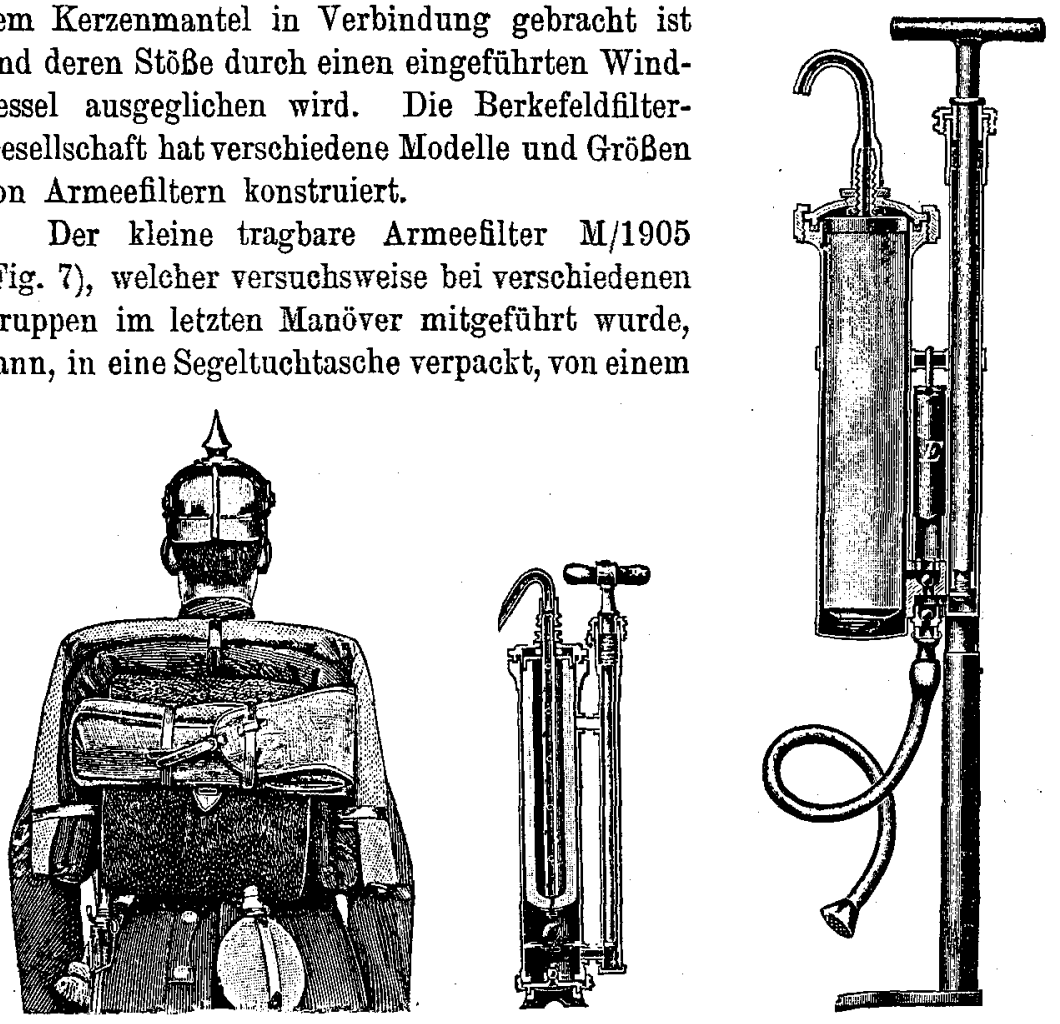

Fig. 7.

Mann an Stelle des Kochgeschirrs getragen werden. Er besteht aus einer Pumpe, aus dem Windkessel, aus der Filterkerze, welche in dem Zylindergehäuse auf einer Feder ruht und durch einen Deckel geschützt ist. An das untere Ende des Pumpenrohres wird ein Verlängerungsrohr zum bequemeren Gebrauch angeschraubt. Das Wasser wird durch einen Schlauch mit Saugstück in die Höhe gesaugt und in das Filtergebäuse sowie in das Innere der Kerze gedrückt. In dieser befindet sich ein durchlöchertes Hetallröhrchen, welches in das im Deckel befindliche Ab- 
laufrohr übergeht. Aus diesem flieBt das filtrierte Wasser aus. Etwas anders angeordnet waren die Filter in Tornisterform, welche eine Zeitlang bei den Truppen des ostasiatischen Expeditionskorps mitgeführt wurden. Das Filter ist an den Deckel des Kastens, der mit dem bei unseren Tornistern verwendeten Kalbsfell überzogen war, befestigt. Durch Drack auf einen Knopf öffnet man den Kasten und schlägt den Deckel nach oben. Der Apparat ist alsdann gebrauchsfertig.

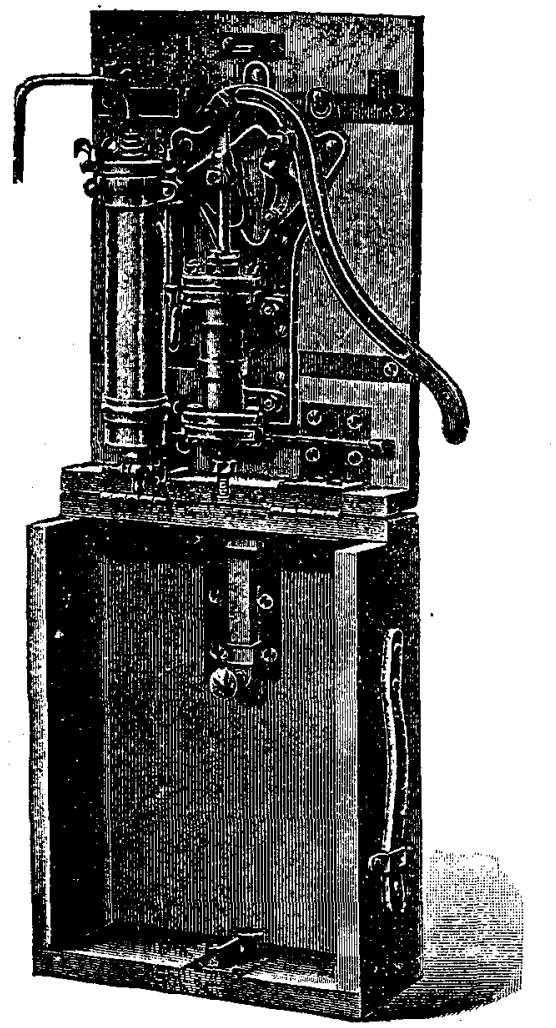

Kleines Feldfilter (F.).

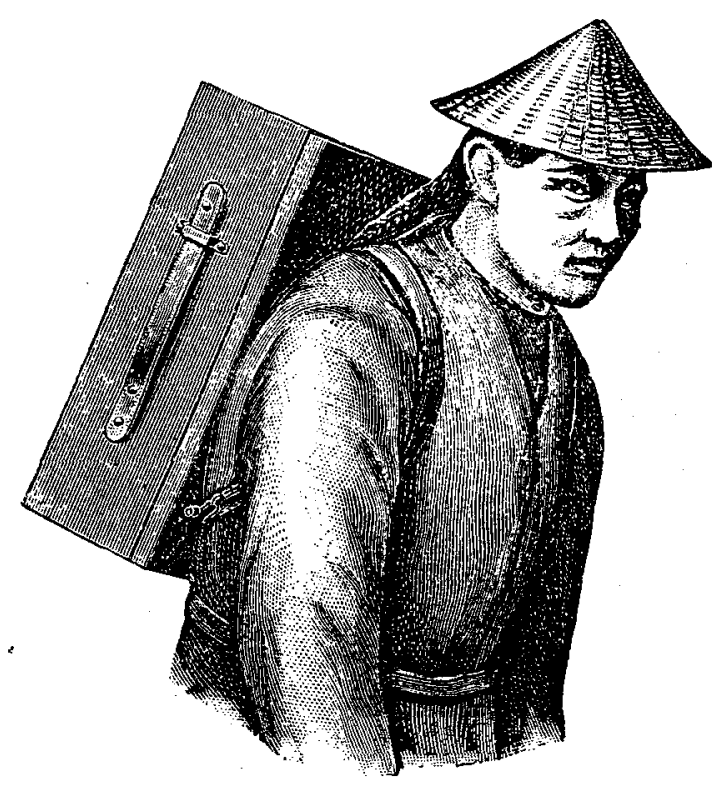

Fig. 8.

Der groBe Armeefilter (Fig. 9 u. 10) besteht aus den gleichen Teilen wie der eben beschriebene kleine; an Stelle einer Kerze sind 9 Filter kranzförmig im Filtergehäuse angeordnet, die ihr Wasser durch einen in der Mitte gelegenen Sammeltrichter in das Ablaufrohr entleeren. Jede Filterkerze kann einzeln ausgeschalten werden. Frisch gereinigt liefert das Filter 15-20 Liter in der Minute, das ist etwa 10 Kochgeschirre voll. Allein die Menge nimmt während des Gebrauchs in dem Maße, als die Poren 
durch die zurūckgehaltenen Keime und Schmutzteilchen verstopft werden, $\mathrm{ab}$; bei stark verunreinigtem Wasser geht schon nach $1 / 4$ Stunde kaum noeh die Hälfte hindurch, nach $1 / 2$ Stunde ist er nicht mehr gebrauchsfähig; die Durchschnittsleistung in der Stunde beträgt bei reinerem Wasser 75-125 Liter. Auch dieser Filter muB bei fortgesetztem Gebrauch täglich sterilisiert werden.

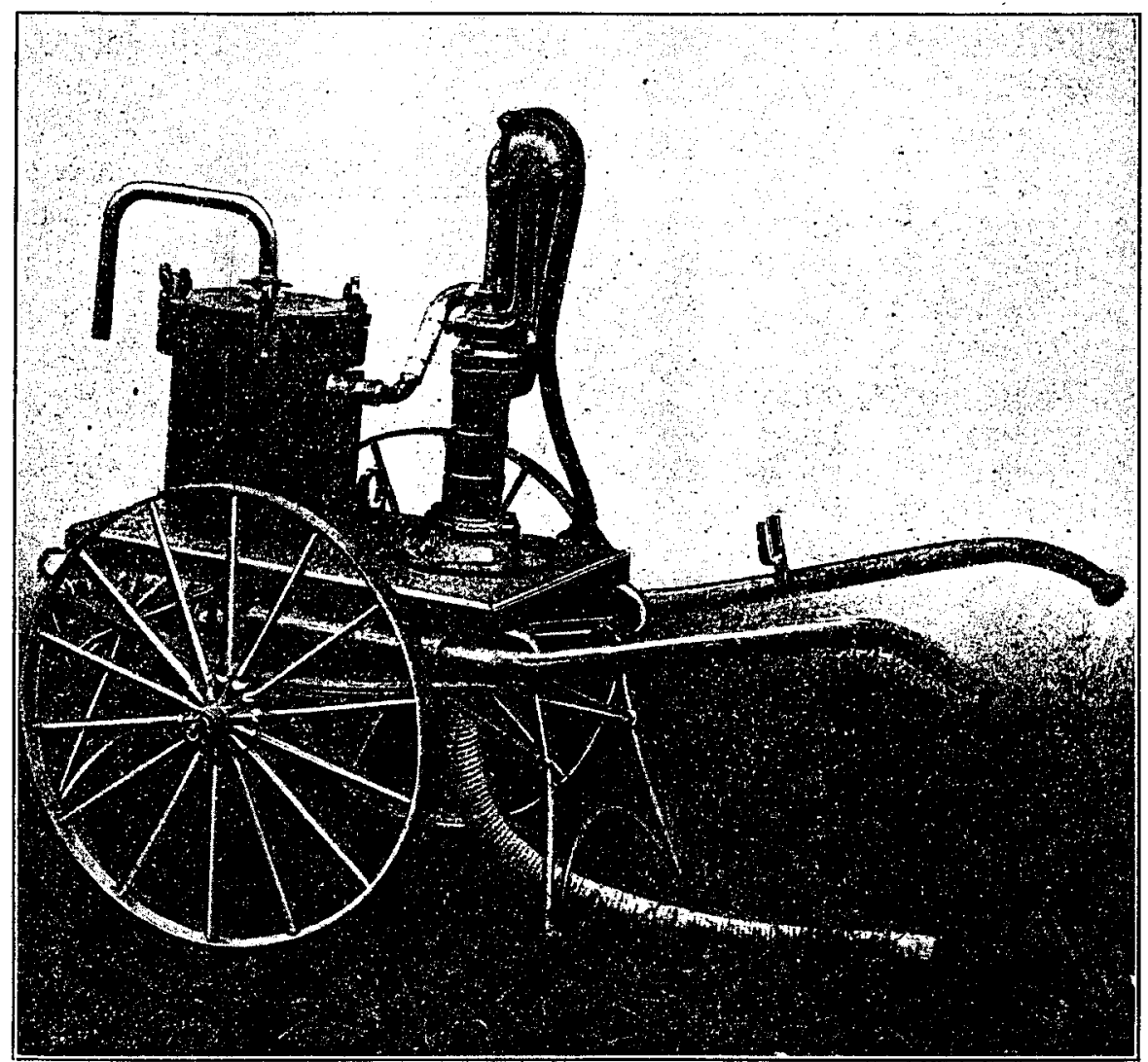

Fig. 9.

Von einer Ausrüstung der Truppen bei. unseren Expeditionen mit dem kleineren Filter wurde bisher Abstand genommen, da die Ergiebigkeit dieser Filter zu klein ist, vor allen Dingen aber, weil die Reinigung und Instandhaltung dieser Apparate mit Schwierigkeiten verknüpft ist, deren Lösung von dem einzelnen Mann ohne sachverständige Beaufsichtigung nicht zu erwarten ist, während eine nicht sachgemäBe Behandlung laus 
den schon angegebenen Gründen geeignet ist ihre Benutzung eher schädlich als nutzbringend zu gestalten. Jedes Bataillon, jede Abteilung, jedes Kavallerie-Regiment war dahingegen mit zwei großen Filtern ausgerüstet. Wenn dieselben auch bei einzelnen Gelegenheiten gute Dienste geleistet haben, so haben die Erfahrungen doch gelehrt, da $B$ die Leistungsfähigkeit auch der groBen Armeefilter auf die Dauer zu unsicher ist, um die Truppen damit auszurüsten. Die erforderliche bakteriologische Kontrolle ihrer Filtrationskraft und die beständige sachgemäBe Überwachung ist bei der marschierenden Truppe unmöglich durchzuführen. ${ }^{1}$

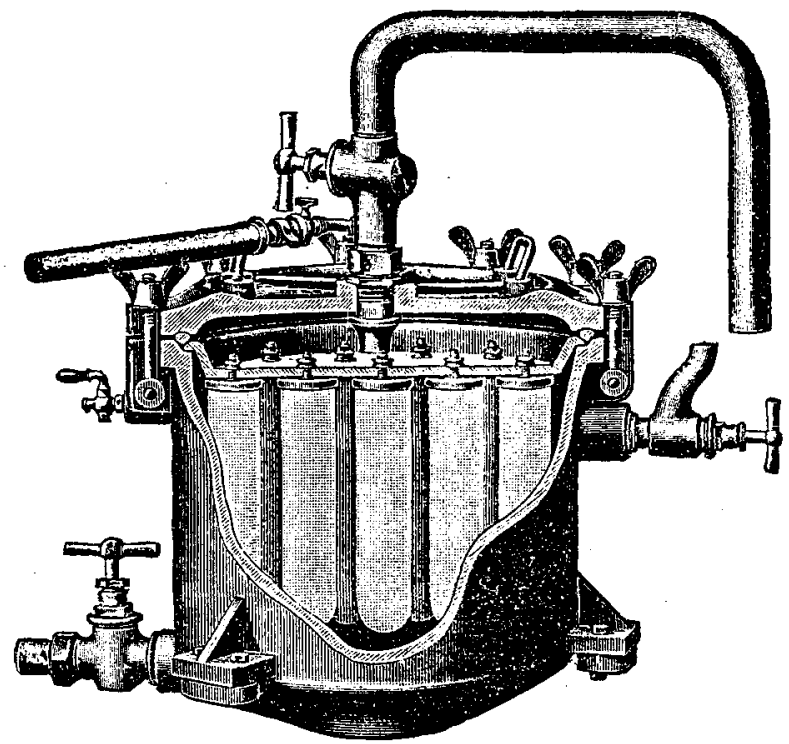

Fig. 10.

Das dritte, fast überall anwendbare Verfahren zur Beschaffung keimfreien Trinkwassers ist das Kochen. Beim Expeditionskorps in China

1 von Leuthold-Gedenkschrift, Bd. I, Musehold and Bischoff: Zur Sicherstellung der Trinkwasserversorgung im Felde, S. 10. Die mit den Berkefeldfiltern während Felddienstïbungen und im Manöver gemachten Versuche, namentlich mit dem Modell 1905, haben im allgemeinen zu einer günstigen Beurteilung geführt, es hat sich gezeigt, daB nicht blob das Sanitätspersonal imstande ist, die Filter richtig zu bedienen. NaturgemäB muß verlangt werden, dab jede einzelne Kerze, bevor sie zur Verwendung kommt, einer Kontrolle ibrer Keimdichtigkeit unterzogen wird, ferner daß für die Bedienung der Filter besonders zuverlässige Leute ausgebildet werden, die dauernd zu kontrollieren sind. 
stand lange Zeit, wie der Sanitätsbericht sagt, der innere Truppendienst unter dem Zeichen des Wasserkochens; so wurden in den Ineisten Quartieren besondere groBe Wasserküchen eingerichtet, in denen Tag und Nacht gekocht wurde, ein Beweis, welche Wichtigkeit das Abkochen erlangt hatte. Etwaige Trübungen wurden durch das Kochen freilich nicht beseitigt; zu diesem Zweck wird man eins der früher erwähnten chemischmechanischen Klärverfahren mit dem Kochen verbinden, wodurch die Fällung der gebildeten Flocken und die Klärung wesentlich beschleunigt wird. Solches Wasser wird nun zwar vollkommen klar und frei ron pathogenen Keimen sein, bedarf aber lange Zeit, um abzukühlen. Erfahrungsgemäß trinken aber durstende Mannschaften viel lieber das trübe

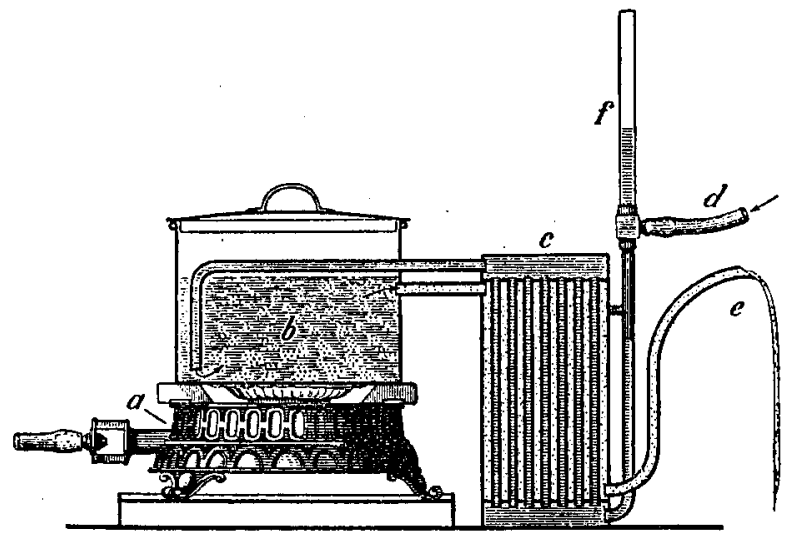

Fig. 11.

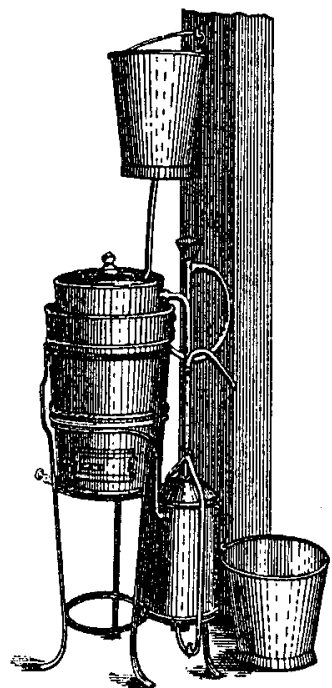

Fig. 12. Sehippecanew.

oder bloß verdächtige, aber kühle Wasser, als laues gekochtes, zumal wenn sie erst $11 / 4$ Stunde darauf warten müssen. Schneller liefern abgekochtes und gekühltes Wasser die Apparate von Siemens und Schuppmann, bei denen durch eine zweckmäßige Anordnung das zugeführte Rohwasser das abfließende gekochte Wasser nach dem Prinzip des Gegenstroms abkühlt. Bei diesen Apparaten ist im wesentlichen ein Kochkessel und eine Kühlvorrichtung vorhanden. Das aus dem $1-2^{\mathrm{m}}$ hoch gehängten Eimer oder Segeltuchbeutel zuflieBende Wasser strömt bei dem Siemensschen Apparat (Fig. 11) durch Röhren, beim Sch up p mann schen Apparat (Fig. 12) durch den äußeren Teil einer doppelwandigen Kühlflasche in den Kochkessel und aus diesem durch andere, den zuführenden anliegende 
Röhren, bzw. durch den inneren Teil der Kühlflasche zurück und etwa $10-15^{\circ}$ wärmer als das Rohwasser aus. Beide Apparate haben den Nachteil, dab das Wasser vor Einlauf in den Apparat geklärt sein muB, $\mathrm{daB}$ das sterilisierte Wasser den faden Geschmack des gekochten Wassers hat, und dab die gelieferte Menge - 40 Liter in der Stunde - für die Zwecke des Heeres nicht ausreichend ist. Nach den bei unseren Expeditionen gemachten Erfahrungen können die Apparate für die auf dem Marsche befindlichen Truppen als nicht sehr geeignet bezeichnet werden. Auf Anregung der Königlichen Medizinalabteilung stellte die Firma Rietschel \& Henneberg einen Wassersterilisator her, welcher die Mängel der vorigen Apparate beseitigte. Die Bedingungen, welche ron seiten der Medizinalabteilung gestellt waren, waren:

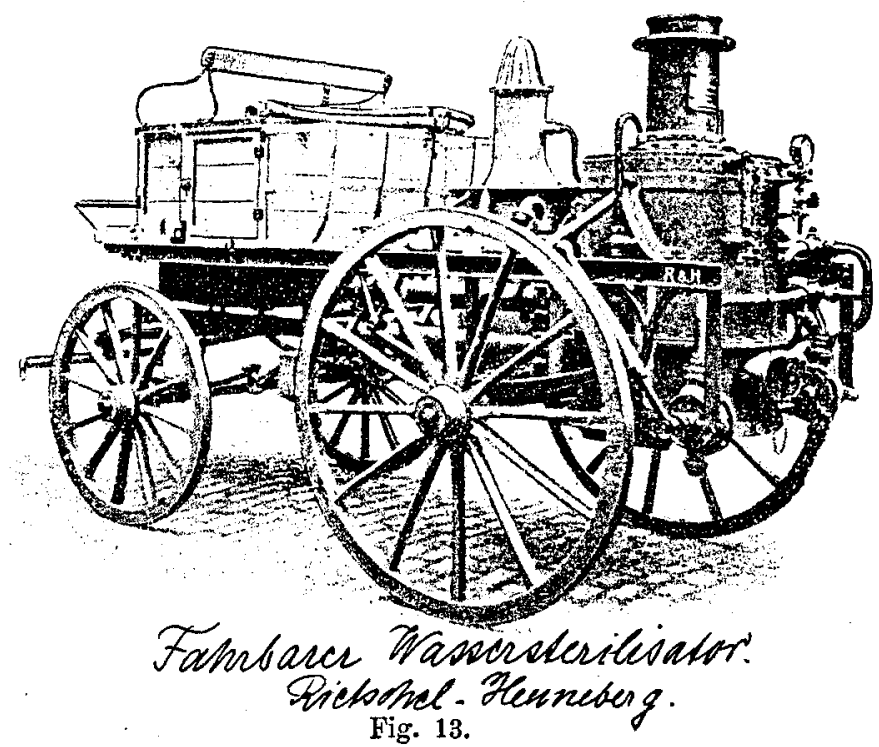

1. Normale Lieferung an Trinkwasser 300 Liter in der Stunde.

2. Absolute Sterilisation des Wassers.

3. Höchsttemperatur des gewonnenen Wassers nicht mehr als $5^{0}$ über der Eintrittstemperatur.

4. Reinigung des Wassers von erdigen oder dergleichen Beimengungen.

5. Vermischung des sterilen Wassers mit Luft.

6. Leicht zu bewerkstelligende Reinigung von Kesselstein und Schlamm.

7. Möglichkeit vor Beginn der Trinkwasserbereitung alle mit diesem in Berührung kommende Teile zu sterilisieren.

8. Maximalgewicht $1300^{\mathrm{kg}}$. 
9. Konstruktion des Wagengestells in der für die Feldgerăte vorgeschriebenen Form.

Der all diese Anforderungen erfüllende Apparat der erwähnten Firma hat sich sowohl bei der Lieferung des Wassers für das Kaiserliche Hauptquartier im Lager von Spengawken während der Herbstübungen 1902 wie auch bei der Expedition in China bewährt. Das MIodell 1904 dieses Apparates (Fig. 13) besteht im wesentlichen aus: 1. dem Kessel zur Erhitzung des Wassers, 2. dem Kühler, 3. dem Filter zur Entfernung grober Unreinigkeiten und zur Wiederbelüftung des Wassers.

Das Wasser wird durch zwei Flügelpumpen mittels eines mit Saugkorb versehenen Schlauches in die Kühlröhren und in den Kessel getrieben. Der Kessel, welcher durch jeden beliebigen festen oder gasförmigen Brennstoff geheizt werden kann, hat die Aufgabe, die Temperatur des ihm zugeführten Rohwassers auf $105-119^{\circ} \mathrm{C}$ zu erhöhen, und arbeitet demgemäß mit einer Spannung ron 0.3 bis 0.5 Atmosphären. Die Gewißheit, daB tatsächlich jeder Tropfen die erforderliche Temperatur angenommen haben muB, bevor er den Kessel verläBt, wird nun je nach der Art des Betriebes, ob kontinuierlich oder intermittierend, auf verschiedene Weise erreicht. In jedem Kochgefä $B$ treten infolge der Verschiedenheit des spezifischen Gewichts des kalten und warmen

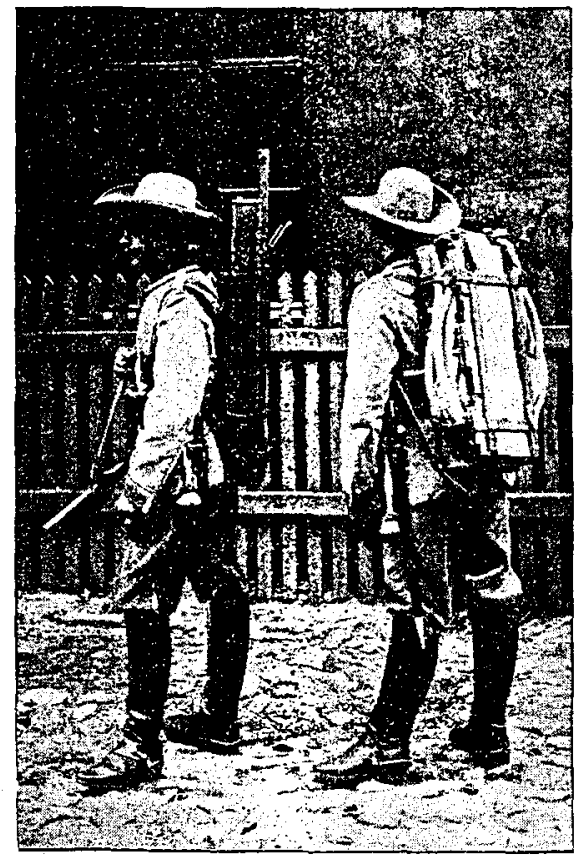

Fig. 14.

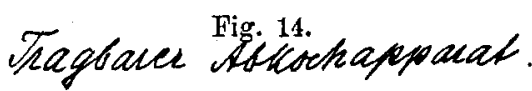
Wassers Zirkulationsbewegungen auf. Besonders heftig werden diese bei Kesseln mit Heiz- oder Siederöhren und durch das Eindringen des stoBweise hineingepumpten Wassers. Man läuft hierbei Gefahr, daB durch diese Strömmungen auch nach längerem Kochen selbst an die heißesten Stellen Wasser getrieben wird, das zur Abtōtung der Keime nicht genūgend erbitzt war. In dem Kessel des fahrbaren Trinkwasserbereiters ist dureh sinnreiche Anbringung einer Rohrschlange, in welche Wasser nur bei 0,3 Atmosphären Druck eintreten kann; dafür gesorgt, daB alles dieses Rohr durchlaufende Wasser einer Temperatur von $105-110^{\circ}$ längere Zeit aus- 


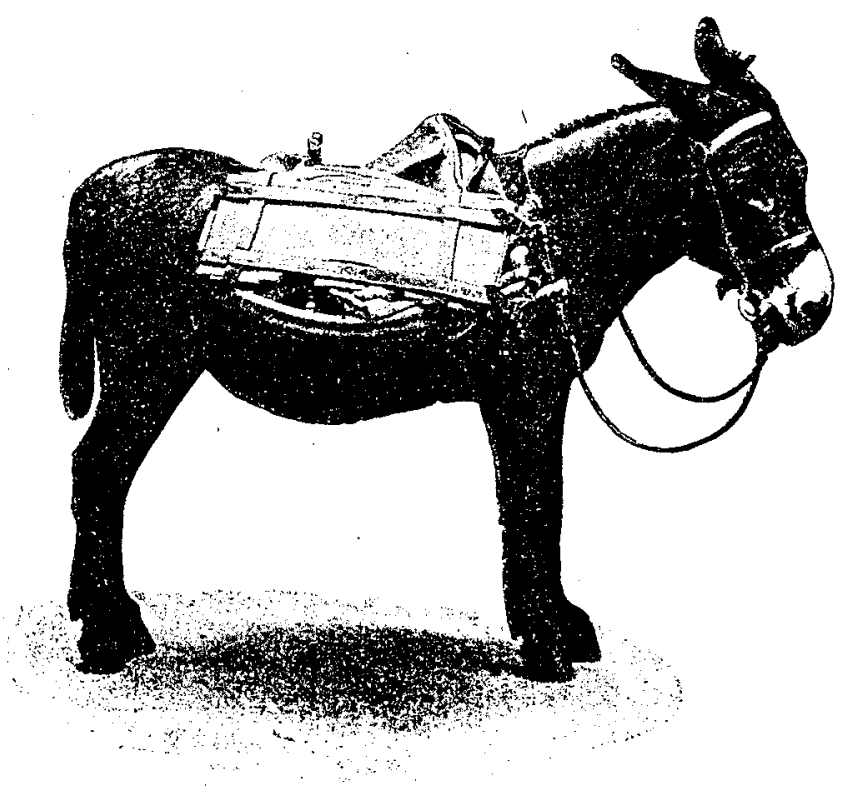

Fig. 15.

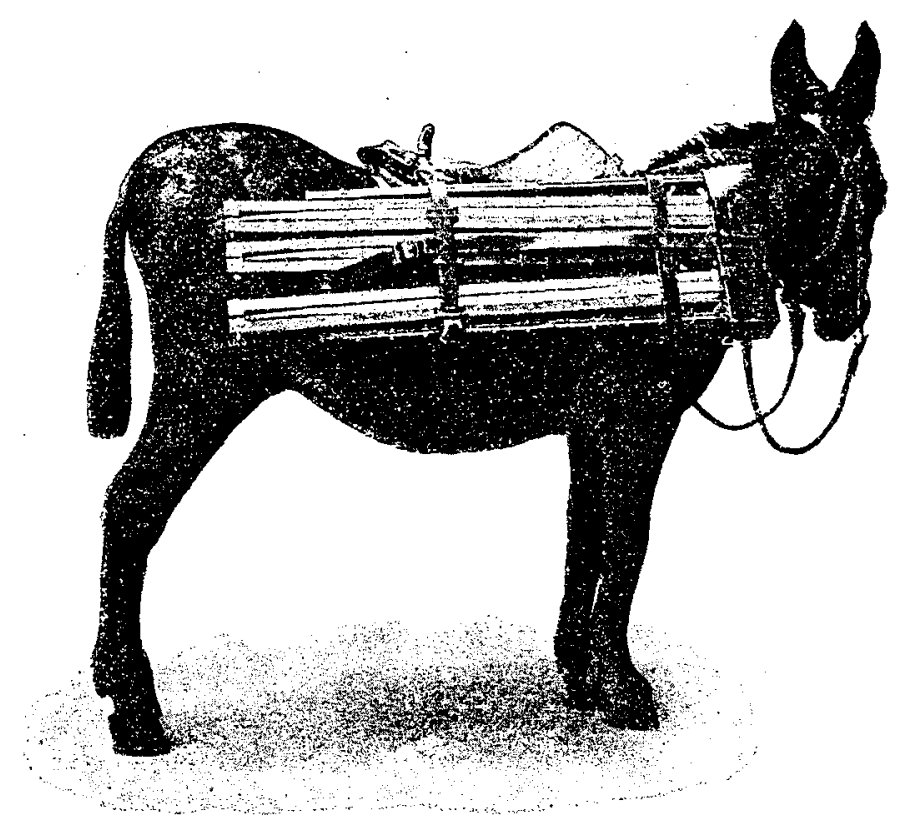

Fig. 16. 
gesetzt gewesen sein muB. Ein in das Wasser bei seinem Austritt hineinreichendes Thermometer gewährt eine ständige Kontrolle. Durch ein Ventil läBt sich vom Kessel aus durch sämtliche Rohrleitungen und Apparate, also durch die ganze Länge des Sterilisierwasserwagens, Dampf leiten. Diese Einrichtung ermöglicht die vollkommene Sterilisation des Wasserweges vor Inbetriebsetzung der Anlage.

Vom Kessel aus gelangt das Wasser in den Kühler. Dieser vermittelt nach dem Prinzip des Gegenstroms den Austausch der Wärme zwischen sterilisiertem und Rohwasser. Er besteht aus 6 Mantelröhren, die au je 3 rechts und links vom Filter angebracht sind, und durch welche

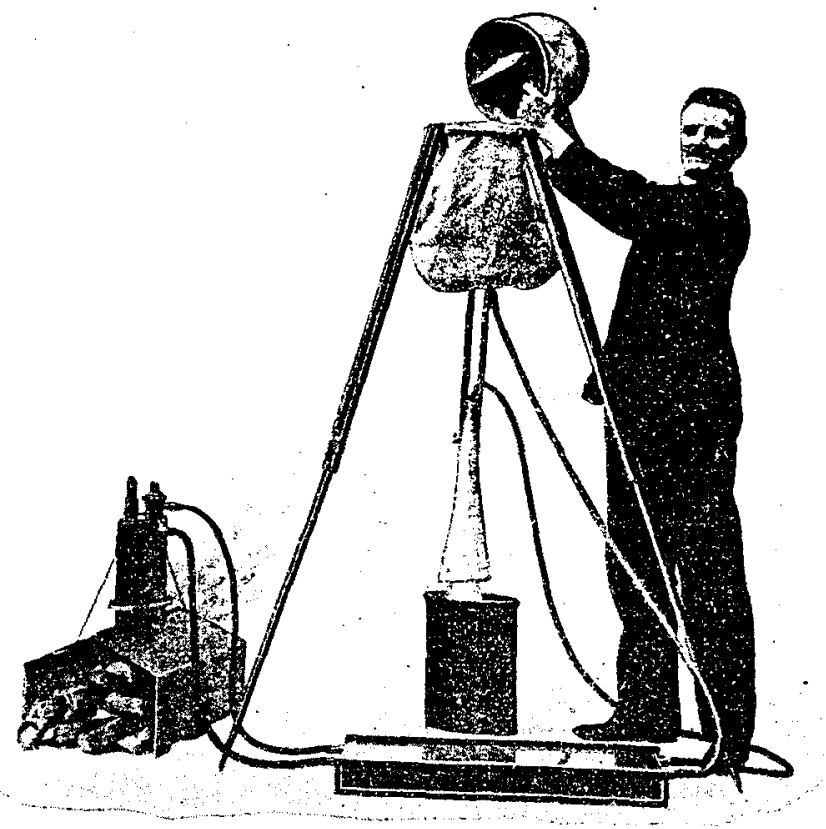

Fig. 17.

in 7 engen Innenröhren das heiße Wasser umspült vom kalten Rohwasser in entgegengesetzter Richtung und stets roneinander getrennt auf das Filter flieBt. Dieses Filter, welches die erdigen oder groben Bestandteile zurückhalten soll, betritt das Wasser durch eine Brause und fällt in Gestalt eines Regens auf das darunter befindliche Filtermaterial, welches aus grober Tierkohle und Bimsstein besteht. Zugleich tritt es auf diese Weise in innige Berührung mit der von außen durch ein keimsicheres Wattefilter in den Apparat eingeführten Luft. Durch die Filtermasse fließt das Wasser langsam in den Vorratsbehälter, aus dem es bis auf $3^{0}$ über das Rohwasser gekühlt, filtriert und gelüftet als gutes Trinkwasser durch 
Hāhne abgelassen werden kann. In der Stunde liefert der Apparat 500 Liter Trinkwasser. ${ }^{1}$

Eingehende Versuche von Proskauer und Schüder haben erwiesen, daß selbst ein so keimhaltiges Wasser, wie das aus dem Spandauer Schifffahrtskanal sicher sterilisiert wird, daB ferner absichtlich mit Typhus-, Ruhr- und Cholerakeimen infiziertes Wasser nach dem Kochen in dem Apparate von den genannten Krankheitserregern sicher befreit wurde.

Durch diesen Apparat ist aber nicht das Bedürfnis kleinerer Truppenabteilungen nach einem guten Trinkwasserbereiter gedeckt. Dieselbe Firma hat deshalb noch einen kleinen; nur $45^{\mathrm{kg}}$ schweren Wassersterilisator konstruiert, welcher sowohl als Paket auf dem Sanitätswagen verladen als auch in zwei Paketen von 22,5 kg Schwere von zwei Mann getragen werden kann (Fig. 14). Die Verpackung des Apparates gestattet auch ihn zu beiden Seiten eines Tragsattels zu befestigen (Fig. 15 u. 16). Dieser tragbare Trinkwasserbereiter setat sich ebenfalls aus den drei Hauptteilen: Kessel, Kühler und Filter zusammen, wozu noch ein Einfüllbeutel mit einem Stativ kommt (Fig. 17).

In dem Einfüllbeutel, welcher aus wasserdichter Leinwand besteht und einen zweiten Beutel aus Filterleinen enthält, wird das Rohwasser von groben Beimengungen befreit. Es flieBt dann zum Kühler, in dem es durch eine besondere Vorrichtung den zur Verlängerung mäanderartig angeordneten Weg zurücklegen muB. Durch den zuführenden Schlauch gelangt das Wasser dann in den oberen Teil des kleinen kupfernen Kesselchens, welcher als Sammelgefäß dient. Er ist von dem unteren Teil des Kessels durch einen Schwimmer getrennt, der nach Füllung des unteren Kesselteils nach oben gedrückt wird. Der untere Kesselteil kann durch beliebiges Material erhitzt werden. Erreicht das Wasser eine

1 von Leuthold-Gedenkschrift, Bd. I, Musehold und Bischoff: Zur Sicherstellung der Trinkwasserversorgung im Felde, S. 16. Das Modell 1905 des fahrbaren Trinkwasserbereiters hat die einfache Kühlerform der tragbaren Apparate übernommen und bietet außerdem den Vorteil, daB die Förderung des Wassers nicht mehr durch Pumpen mit Handbetrieb erfolgen muB, sondern daB eine Dampfpumpe für' die Speisung des Apparates vorgesehen ist. Der Betrieb bei diesem Apparate regelt sich folgendermaBen: Beim Beginn wird der Kessel mit der Handpumpe gespeist. Ist im Kessel der gewünschte Dampfdruck von 0.5 Atmosphären vorhanden, so wird die Dampfpumpe angestellt. Die Dimensionen des Dampfzylinders sind bei dieser Pumpe wesentlich größer als die des Pumpenzylinders, wodurch es ermöglicht ist, mit einem Dampfdruck von 0.5 Atmosphären das Wasser durch den Kühler in den Kessel gegen den nämlichen Dampfdruck, der für die Pumpe zur Verfügung steht, zu drücken. Bei diesem vollkommensten Wassersterilisationsapparat ist somit infolge Einführung mechanischen Pumpenbetriebes nur noch ein Mann zur Bedienung erforderlich, der die Ventile bedient und das Nachschütten von Heizmaterial besorgt. 
Temperatur von $105^{\circ}$, so wird durch den hierbei entstehenden Dampfdruck von 0,3 Atmosphären ein genau hierauf eingestelltes doppelsitziges Federventil geöffnet, und das sterilisierte Wasser fließt durch den AbfluBschlauch zum Kühler zurück. Gleichzeitig öffnet nun der Schwimmer den Weg für das im oberen Teil befindliche Rohwasser, welehes fast augenblicklich nach unten stürzt. Der Erhitzungsvorgang beginnt nun von neuem. Im Kühler muß das heiße Wasser seinen Weg an der gekühlten Scheidewand wie beim ZufluB zurücklegen, wodurch es bis auf $3^{0}$

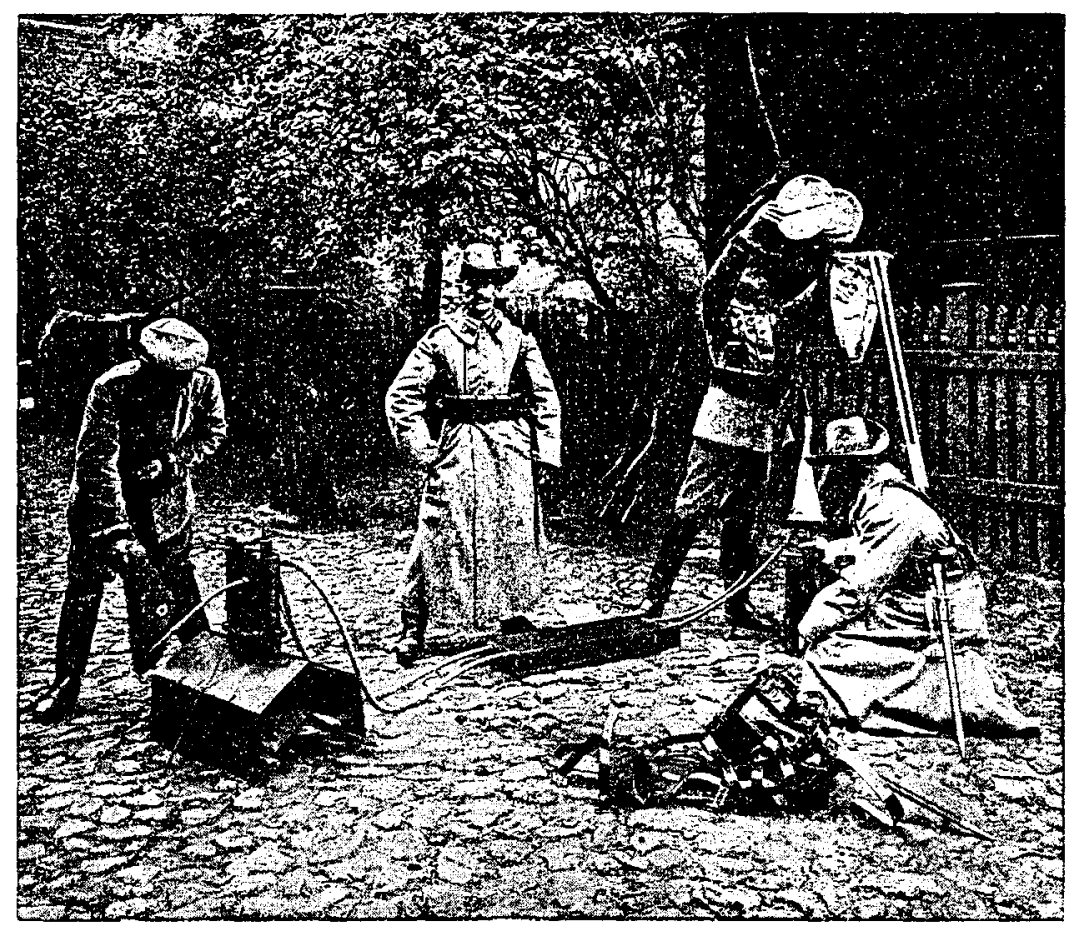

Fig. 18.

über die Temperatur des Rohwassers abgekühlt zum Filter gelangt. Dieses ist aus einem Beutel wasserdichter Leinwand gebildet, der unten die Filterschicht trägt und oben durch Luftzutritt in geeigneter Weise für Lüftung sorgt. Es ist an dem Stativ befestigt, welches den Einfüllbeutel trägt. Aus dem Filter fließt es in ein darunter stehendes Gefäß. Die Bedienung ist eine einfache und beschränkt sich auf Unterhaltung des Feuers und Einfüllung von Wasser (Fig. 18). Der Apparat liefert nach etwa 18 Minuten schon Trinkwasser und in einer Stunde 100 Liter. 
Generaloberarzt Schian sagt in seinem Vortrag über die Trinkwasserversorgung in Afrika, wo diese Apparate Verwendung gefunden haben: „Die fahrbaren Wassersterilisatoren arbeiteten, wenn sie klares Wasser zu kochen hatten, tadellos, ebenso die kleineren Apparate." - Er machte aber die Erfahrung, daB ihr Material für afrikanische Verhältnisse nicht widerstandsfähig genug war; auch war es unmöglich, soviel Apparate den Feldtruppen mitzugeben, daß jede kleine Abteilung einen hatte. Von allen angewandten Verfahren hat sich aber nach seinem Urteil, welches auch durch die Erfahrungen beim Expeditionskorps in China und bei der japanischen Armee bestätigt wird, das Abkochen bewährt.

$O b$ die Möglichkeit in der Zukunft vorliegt, die Truppenteile mit einer hinreichenden Menge Trinkwasserbereiter zu versehen, darüber zu entscheiden bleibt Sache der Militärverwaltung. Die Aufgabe, für den Feldgebrauch geeignete fahr- und tragbare Trinkwasserbereiter zu schaffen, muB als gelöst bezeichnet werden. 


\section{Literatur-Verzeichnis.}

Arbeiten a. d. Kaiserl. Gesundheitsamte. Bd. XIII, XIV, XVII, XIX u. XXI.

Erlwein, Über Trinkwasserreinigung durch Ozon und Ozonwasserwerke. Gesundheit. 1903. Nr. 18.

Derselbe, Trinkwasserreinigung durch Ozon nach dem System von Siemens. Journal für Gasbeleuchtung und Wasserversorgung. XLIV. Nr. 30/31.

Der selbe, Einzelanlagen zur Sterilisation von Trink- u. Industriewasser durch Ozon. Gesundheit. 1906. Bd. III.

Flüg ge, Lehrbuch der Hygiene.

Derselbe, Über die Beziehungen zwischen Flußwasser und Grundwasser in Breslau, nebst kritischen Bemerkangen über die Leistungsfähigkeit der chemischen Trinkwasseranalyse. Diese Zeitschrift. Bd. XXII und XXIII.

Derselbe, Hygienische Beurteilung von Trink- und Nutzwasser. Deutscle Vierteljahrsschrift für öffentliche Gesundheitspflege. Bd. XXVIII.

Gärtner, Die Quellen in ihrer Beziehung zum Grundwasser und Typhus. Klinisches Jahrbuch. Bd. IX.

Derselbe, Zur Hygiene der Wasserversorgung. Journal für Gasbeleuchtung und Wasserversorgung. XLVII. Nr. 34/35.

Hiller, Gesundheitspflege des Heeres.

Hünermann und Deiter, Über die Desinfektion des Trinkwassers mit Natr. hypochlorit. Deutsche med. Wochenschrift. 1901. Nr. 24.

Kir eh ner, Militärgesundheitslehre. Bd. IX

Derselbe, Ernährung und Trinkwasserversorgung im Felde. Klin. Jahrbuch. 1897.

Kraschutzki, Trinkwasserversorgung. Deutsche militäräratliche Zeitschrift.

Kretschmar, Über Beschaffung vou gesundem Trinkwasser im Lager und während des Marsches mit Rücksicht auf die Filtrationsmethoden. Der Militärarzt. 1894. Nr. 21-24. - 1895. Nr. 1-6.

Kriegs-Sanitätsordnung.

Krüger, Die physikalische Einwirkung von Sinkstoffen anf die im Wasser befindlichen Mikroorganismen. Diese Zeitschrift. Bd. VII.

Mez, Mikroskopische Wasseranalyse.

Mitteilungen aus der wissensehaftlichen Versammlung der Militärärzte in Danzig. Deutsche militärärztliehe Zeitschrift. 1906.

Morgenroth and Weigt, Bericht über die Wasserversorgang in und um Tientsin. Hygienische Rundschau. 1901. Nr. 16.

Morgenroth und Bassenge, Bericht über die im bakteriologischen und chemischen Laboratorium zu Tientsin in der Zeit vom 1. X. 1900 bis 1. III. 1901 ausgeführten Arbeiten. Deutsche militärärztliche Zeitschrift. 1901. 
Ohlmüller und Prall, Behandlung des Trinkwassers mit Ozon. Arbeiten aus dem Kaiserl. Gesundheitsamte. Bd. XVIII.

Ohlmüller, Reinigung des Trinkwassers durch Ozon. Deutsche Vierteljahrssehrift für öffentl. Gesundheitspflege. 1904. Bd. XXXVI.

Plagge, Untersuchungen über Wasserfilter. Veröffentlichungen aus dem Gebiete des Militär-Sanitätswesens. Hft. 9.

Proskauer n. Schüder, Über die Abtötung pathogener Bakterien im Wasser mittels Ozon. Diese Zeitschrift. Bd. XLI.

Dieselben, Weitere Versuche mit dem Ozon als Wassersterilisationsmittel im Wiesbadener Ozonwasserwerk. Ebenda. Bd. XIII.

Pfeiffer und Proskauer, Enzyklopädie für Hygiene, Wisser (Gärtner), Wassersterilisation ( $\mathrm{Sch}$ ĩ der).

Rietschel und Henneberg, Trinkwasserbereiter. Katalog für die Weltausstellung in St. Louis 7904.

Sanitätsbericht über das Kaiserl. ostasiatische Expeditionskorps für den Berichtsraum vom 1. VII. 1900 bis 30. VI. 1901.

Schian, Über die Trinkwasserversorgung in Afrika. Deutsche militärärztliche Zeitschrift. 1905. Hft. 11.

Schmiedicke, Über Brunnenanlagen bei Truppenübungen. Ebenda. 1906. Hft.2.

Schüder, Über das Schumburgsche Verfahren der Wasserreinigung mittels Brom. Diese Zeitschrift. Bd. XXXVII.

Derselbe, Über das Hünermannsche Verfahren der Wasserdesinfektion nebst Bemerkungen über die bei der Prüfung derartiger Desinfektionsmittel anzuwendenden Untersuchungsmethoden. Ebenda. Bd. XXXIX.

Dersel be, Entgegnung anf die Schu m burgsche drbeit: ,Das Wasserreinigungsverfahren mit Brom“. Ebenda. Bd. XXXIX a. XI.

Schüder und Proskauer, Versuche mit dem fahrbaren Trinkwasserbereiter von Rietschel und Henneberg. Ebenda. Bd. XL.

Schumburg and Plagge, Beiträge zur Frage der Trinkwasserbereitung. Veröffentlichungen aus dem Gebiete des Militär-Sanitätswesens. Hft. 15.

Schumburg, Ein neues Verfahren zur Herstellung keinfreien Wassers. Deutscle med. Wochenschrift. 1897. Nr. 10.

Derselbe, Verfahren der Wasserreinigung durch Bromzusatz. Deutsche militärärztliche Zeitschrift. 1897. Hft. 7.

Derselbe, Das Wasserreinigungsverfahren mit Brom. Diese Zeitschr. Bd.XXXIX.

Seaman, The real triumph of Japan or the conquest of the silent foe. Nach dem Referat in der Deutschen militärärztlichen Zeitschrift. 1906. Hft. 1.

Traube, Einfaches Verfahren, Wasser in großen Mengen keimfrei zu machen. Diese Zeitschrift. Bd. XVI.

Uffelmann, Handbuch für Hygiene.

Weyl, Keimfreies Trinkwasser mittels Ozon. Centralblatt für Bakteriologie. Abt. I. Bd. XXVI.

Derselbe, Über die Verwendung von Ozon zur Gewinnang keimfreien Trinkwassers. Journal für Gasbeleuchtung und Wasserversorgung. XLII. Nr. 48/49. 